\title{
RESIDUAL A POSTERIORI ERROR ESTIMATORS FOR CONTACT PROBLEMS IN ELASTICITY
}

\author{
PATRICK Hild $^{1}$ AND SERGE NicAise ${ }^{2}$
}

\begin{abstract}
This paper is concerned with the unilateral contact problem in linear elasticity. We define two a posteriori error estimators of residual type to evaluate the accuracy of the mixed finite element approximation of the contact problem. Upper and lower bounds of the discretization error are proved for both estimators and several computations are performed to illustrate the theoretical results.
\end{abstract}

Mathematics Subject Classification. 65N30, 74M15.

Received September 26, 2006. Revised April 17, 2007.

\section{INTRODUCTION}

The finite element method is currently used in the numerical realization of contact problems occurring in several engineering applications (see $[18,19,24,25,30])$. An important task consists of evaluating numerically the quality of the finite element computations by using a posteriori error estimators. There are two important difficulties in developing such tools for contact problems in elasticity: the first one comes from the inequality (unilateral) conditions in the model and the second one is due to the location of these inequality conditions which hold on (a part of) the boundary. For the linear elasticity system with standard boundary conditions (leading to a variational identity), many different approaches leading to various error estimators have been developed and a review of the different a posteriori error estimators can be found in [29]. Some of these approaches have been chosen and studied for frictionless or frictional unilateral contact problems, in particular in [7,26,31] (residual approach using a penalization of the contact condition or the normal compliance law), in [10,11] (equilibrated residual method) and finally in [14] (residual approach for BEM-discretizations).

Besides, let us mention that many studies dealing with residual estimators for scalar variational inequality problems of the first kind have been achieved in other contexts than elasticity. In particular a great effort was devoted to the obstacle problem (see e.g., $[6,8,27]$ and the references therein). Moreover a residual type estimator for the Signorini problem in its standard formulation can be found in [21] (note that the Signorini problem could be seen as a simplification in the scalar case of the unilateral contact model). For residual estimators dealing with variational inequalities of the second kind we refer the reader to [5] and the references therein. We recall that a variational inequality of the first (resp. second) kind is of the form: $u \in C, a(u, v-u) \geq L(v-u), \forall v \in C$ (resp. $u \in X, a(u, v-u)+j(v)-j(u) \geq L(v-u), \forall v \in X)$ where $X$ is an Hilbert space, $C \subset X$ is a nonempty closed convex set, $a(.,$.$) is bilinear, X$-elliptic and continuous on $X \times X, L($.$) is linear and continuous on X$, $j($.$) is proper convex and lower semi continuous on X$ (with values in $\mathbb{R} \cup\{+\infty\}$ ). More details concerning variational inequalities of the first or second kind can be found in e.g., $[2,17]$.

Keywords and phrases. Mixed finite element method, a posteriori error estimates, residuals, unilateral contact.

1 Université de Franche-Comté, Laboratoire de Mathématiques de Besançon, CNRS UMR 6623, 16 route de Gray, 25030 Besançon, France. hild@math.univ-fcomte.fr

2 Université de Valenciennes et du Hainaut Cambrésis, MACS, ISTV, 59313 Valenciennes Cedex 9, France.

snicaise@univ-valenciennes.fr

(c) EDP Sciences, SMAI 2007 Article published by EDP Sciences and available at http://www.esaim-m2an.org or http://dx.doi.org/10.1051/m2an:2007045 
In the present work we are interested in developing residual estimators for the two-dimensional unilateral contact model in linear elasticity. This problem can be written as a variational inequality of the first kind but also (among others) as a mixed formulation where the unknowns are the displacement field and the contact pressure.

The paper is organized as follows. In Section 2 we introduce the equations modelling the frictionless unilateral contact problem between an elastic body and a rigid foundation. We write the problem using a mixed formulation where the unknowns are the displacement field in the body and the pressure on the contact area. In the third section, we choose a classical discretization involving continuous finite elements of degree one and continuous piecewise affine multipliers on the contact zone. Section 4 is concerned with the study of a first residual estimator which can be seen as the natural one arising from the discrete problem. We obtain both global upper and local lower bounds of the error. In Section 5 we consider a second estimator resulting from another discrete model where the displacement field is the same as in the first model but where the multiplier is modified. The main novelty of the second discrete model is that the multipliers have a constant sign. As in Section 4, we obtain global upper and local lower bounds of the error. Finally in Section 6 we implement both estimators and we compare them on several examples.

Finally we introduce some useful notation and several functional spaces. In what follows, bold letters like $\mathbf{u}, \mathbf{v}$, indicate vector valued quantities, while the capital ones $(e . g ., \mathbf{V}, \mathbf{K}, \ldots)$ represent functional sets involving vector fields.

As usual, we denote by $\left(L^{2}(.)\right)^{d}$ and by $\left(H^{s}(.)\right)^{d}, s \geq 0, d=1,2$ the Lebesgue and Sobolev spaces in one and two space dimensions (see [1]). The usual norm of $\left(H^{s}(D)\right)^{d}$ is denoted by $\|\cdot\|_{s, D}$ and we keep the same notation when $d=1$ or $d=2$. For shortness the $\left(L^{2}(D)\right)^{d}$-norm will be denoted by $\|\cdot\|_{D}$ when $d=1$ or $d=2$. In the sequel the symbol $|\cdot|$ will denote either the Euclidean norm in $\mathbb{R}^{2}$, or the length of a line segment, or the area of a plane domain. Finally the notation $a \lesssim b$ means here and below that there exists a positive constant $C$ independent of $a$ and $b$ (and of the meshsize of the triangulation) such that $a \leq C b$. The notation $a \sim b$ means that $a \lesssim b$ and $b \lesssim a$ hold simultaneously.

\section{The Unilateral CONTACT PROBLEM IN ELASTICITY}

Let $\Omega$ represent an elastic body in $\mathbb{R}^{2}$ where plane strain assumptions are assumed. The boundary $\partial \Omega$ is supposed to be polygonal, i.e., it is the union of a finite number of linear segments. Moreover we suppose that the boundary consists in three nonoverlapping parts $\Gamma_{D}, \Gamma_{N}$ and $\Gamma_{C}$ with meas $\left(\Gamma_{D}\right)>0$ and meas $\left(\Gamma_{C}\right)>0$. The normal unit outward vector on $\partial \Omega$ is denoted $\mathbf{n}=\left(n_{1}, n_{2}\right)$ and we choose as unit tangential vector $\mathbf{t}=\left(-n_{2}, n_{1}\right)$. In its initial configuration, the body is in contact on $\Gamma_{C}$ and we suppose that the unknown final contact zone after deformation will be included in $\Gamma_{C}$. The body is clamped on $\Gamma_{D}$ for the sake of simplicity. It is subjected to volume forces $\mathbf{f}=\left(f_{1}, f_{2}\right) \in\left(L^{2}(\Omega)\right)^{2}$ and to surface forces $\mathbf{g}=\left(g_{1}, g_{2}\right) \in\left(L^{2}\left(\Gamma_{N}\right)\right)^{2}$.

The unilateral contact problem in elasticity consists in finding the displacement field $\mathbf{u}: \Omega \rightarrow \mathbb{R}^{2}$ verifying the equations and conditions (1)-(6):

$$
\operatorname{div} \boldsymbol{\sigma}(\mathbf{u})+\mathbf{f}=\mathbf{0} \quad \text { in } \Omega
$$

where div denotes the divergence operator of tensor valued functions and $\boldsymbol{\sigma}=\left(\sigma_{i j}\right), 1 \leq i, j \leq 2$, stands for the stress tensor field. The latter is obtained from the displacement field by the constitutive law of linear elasticity

$$
\boldsymbol{\sigma}(\mathbf{u})=\mathbf{A} \varepsilon(\mathbf{u}) \quad \text { in } \Omega
$$

where $\mathbf{A}$ is a fourth order symmetric and elliptic tensor and $\varepsilon(\mathbf{v})=\left(\nabla \mathbf{v}+{ }^{t} \nabla \mathbf{v}\right) / 2$ represents the linearized strain tensor field. On $\Gamma_{D}$ and $\Gamma_{N}$, the conditions are as follows:

$$
\begin{aligned}
\mathbf{u} & =\mathbf{0} & & \text { on } \Gamma_{D}, \\
\boldsymbol{\sigma}(\mathbf{u}) \mathbf{n} & =\mathbf{g} & & \text { on } \Gamma_{N} .
\end{aligned}
$$


Afterwards we choose the following notation for any displacement field $\mathbf{v}$ and for any density of surface forces $\boldsymbol{\sigma}(\mathbf{v}) \mathbf{n}$ defined on $\partial \Omega$ :

$$
\mathbf{v}=v_{n} \mathbf{n}+v_{t} \mathbf{t} \quad \text { and } \quad \boldsymbol{\sigma}(\mathbf{v}) \mathbf{n}=\sigma_{n}(\mathbf{v}) \mathbf{n}+\sigma_{t}(\mathbf{v}) \mathbf{t} .
$$

The conditions modelling unilateral contact on $\Gamma_{C}$ are (see e.g., $\left.[13,15,16]\right)$ :

$$
u_{n} \leq 0, \quad \sigma_{n}(\mathbf{u}) \leq 0, \quad \sigma_{n}(\mathbf{u}) u_{n}=0 .
$$

Finally the condition

$$
\sigma_{t}(\mathbf{u})=0
$$

on $\Gamma_{C}$ means that friction is omitted.

In order to derive the variational formulation of (1)-(6), we consider the Hilbert space

$$
H_{\Gamma_{D}}^{1}(\Omega)=\left\{v \in H^{1}(\Omega): \quad v=0 \text { on } \Gamma_{D}\right\}
$$

equipped with the $H^{1}(\Omega)$-norm. We further use the Hilbert space

$$
\mathbf{V}=\left(H_{\Gamma_{D}}^{1}(\Omega)\right)^{2} .
$$

For our next uses, we introduce the trace space $H^{\frac{1}{2}}\left(\Gamma_{C}\right)$ as follows:

$$
H^{\frac{1}{2}}\left(\Gamma_{C}\right)=\left\{\phi \in L^{2}\left(\Gamma_{C}\right): \exists u \in H_{\Gamma_{D}}^{1}(\Omega) \text { such that } \phi=\gamma u \text { on } \Gamma_{C}\right\},
$$

equipped with the norm

$$
\|\phi\|_{\frac{1}{2}, \Gamma_{C}}=\inf _{u \in H_{\Gamma_{D}}^{1}(\Omega): \phi=\gamma u}\|u\|_{1, \Omega},
$$

where $\gamma$ is the standard trace operator from $H^{1}(\Omega)$ to $H^{\frac{1}{2}}(\partial \Omega)$ (see [1]). The topological dual space of $H^{\frac{1}{2}}\left(\Gamma_{C}\right.$ ) will be denoted by $H^{-\frac{1}{2}}\left(\Gamma_{C}\right)$, whose norm is

$$
\|\psi\|_{-\frac{1}{2}, \Gamma_{C}}=\sup _{\phi \in H^{\frac{1}{2}}\left(\Gamma_{C}\right)} \frac{\langle\psi, \phi\rangle_{-\frac{1}{2}, \frac{1}{2}, \Gamma_{C}}}{\|\phi\|_{\frac{1}{2}, \Gamma_{C}}},
$$

where the notation $\langle., .\rangle_{-\frac{1}{2}, \frac{1}{2}, \Gamma_{C}}$ represents the duality pairing between $H^{-\frac{1}{2}}\left(\Gamma_{C}\right)$ and $H^{\frac{1}{2}}\left(\Gamma_{C}\right)$.

The forthcoming mixed variational formulation uses the following convex cone of multipliers on $\Gamma_{C}$

$$
M=\left\{\mu \in H^{-\frac{1}{2}}\left(\Gamma_{C}\right):\langle\mu, \psi\rangle_{-\frac{1}{2}, \frac{1}{2}, \Gamma_{C}} \geq 0 \text { for all } \psi \in H^{\frac{1}{2}}\left(\Gamma_{C}\right), \psi \geq 0 \text { a.e. on } \Gamma_{C}\right\} .
$$

Define

$$
\begin{aligned}
a(\mathbf{u}, \mathbf{v}) & =\int_{\Omega} \boldsymbol{\sigma}(\mathbf{u}): \varepsilon(\mathbf{v}) \mathrm{d} \Omega, \quad b(\mu, \mathbf{v})=\left\langle\mu, v_{n}\right\rangle_{-\frac{1}{2}, \frac{1}{2}, \Gamma_{C}}, \\
L(\mathbf{v}) & =\int_{\Omega} \mathbf{f} \cdot \mathbf{v} \mathrm{d} \Omega+\int_{\Gamma_{N}} \mathbf{g} \cdot \mathbf{v} \mathrm{d} \Gamma
\end{aligned}
$$

for any $\mathbf{u}$ and $\mathbf{v}$ in $\mathbf{V}$ and $\mu$ in $H^{-\frac{1}{2}}\left(\Gamma_{C}\right)$. 
The mixed formulation of the unilateral contact problem without friction (1)-(6) consists then in finding $\mathbf{u} \in \mathbf{V}$ and $\lambda \in M$ such that:

$$
\left\{\begin{array}{l}
a(\mathbf{u}, \mathbf{v})+b(\lambda, \mathbf{v})=L(\mathbf{v}), \quad \forall \mathbf{v} \in \mathbf{V}, \\
b(\mu-\lambda, \mathbf{u}) \leq 0, \quad \forall \mu \in M
\end{array}\right.
$$

An equivalent formulation of (7) consists in finding $(\lambda, \mathbf{u}) \in M \times \mathbf{V}$ satisfying

$$
\mathcal{L}(\mu, \mathbf{u}) \leq \mathcal{L}(\lambda, \mathbf{u}) \leq \mathcal{L}(\lambda, \mathbf{v}), \quad \forall \mathbf{v} \in \mathbf{V}, \forall \mu \in M
$$

where $\mathcal{L}(\mu, \mathbf{v})=\frac{1}{2} a(\mathbf{v}, \mathbf{v})-L(\mathbf{v})+b(\mu, \mathbf{v})$. Another classical weak formulation of problem (1)-(6) is a variational inequality: find $\mathbf{u}$ such that

$$
\mathbf{u} \in \mathbf{K}, \quad a(\mathbf{u}, \mathbf{v}-\mathbf{u}) \geq L(\mathbf{v}-\mathbf{u}), \quad \forall \mathbf{v} \in \mathbf{K}
$$

where $\mathbf{K}$ denotes the closed convex cone of admissible displacement fields satisfying the non-penetration conditions:

$$
\mathbf{K}=\left\{\mathbf{v} \in \mathbf{V}: \quad v_{n} \leq 0 \text { on } \Gamma_{C}\right\} .
$$

The existence and uniqueness of $(\lambda, \mathbf{u})$ solution to (7) has been stated in [19]. Moreover, the first argument $\mathbf{u}$ solution to (7) is also the unique solution of problem (8) and $\lambda=-\sigma_{n}(\mathbf{u})$.

\section{Mixed Finite ELEMENT APPROXIMATION}

We approximate this problem by a standard finite element method. Namely we fix a family of meshes $T_{h}, h>0$, regular in Ciarlet's sense [9], made of closed triangles. For $K \in T_{h}$ we recall that $h_{K}$ is the diameter of $K$ and $h=\max _{K \in T_{h}} h_{K}$. The regularity of the mesh implies in particular that for any edge $E$ of $K$ one has $h_{E}=|E| \sim h_{K}$.

Let us define $E_{h}$ (resp. $\mathcal{N}_{h}$ ) as the set of edges (resp. nodes) of the triangulation and set $E_{h}^{\text {int }}=\{E \in$ $\left.E_{h}: E \subset \Omega\right\}$ the set of interior edges of $T_{h}$ (the edges are supposed to be relatively open). We denote by $E_{h}^{N}=\left\{E \in E_{h}: E \subset \Gamma_{N}\right\}$ the set of exterior edges included into the part of the boundary where we impose Neumann conditions, and similarly $E_{h}^{C}=\left\{E \in E_{h}: E \subset \Gamma_{C}\right\}$ is the set of exterior edges included into the part of the boundary where we impose unilateral contact conditions. Set $\mathcal{N}_{h}^{D}=\mathcal{N}_{h} \cap \overline{\Gamma_{D}}$ (note that the extreme nodes of $\overline{\Gamma_{D}}$ belong to $\mathcal{N}_{h}^{D}$ ). Let $\mathcal{S}$ denote the set of vertices of $\Omega$ and denote by $\mathcal{N}_{h}^{N C}$ the set of nodes which belong to $\overline{\Gamma_{C}} \cap \overline{\Gamma_{N}}$ and by $\mathcal{N}_{h}^{C C}$ the nodes belonging to $\Gamma_{C} \cap \mathcal{S}$. Set finally $\mathcal{N}_{h}^{C}=\left(\mathcal{N}_{h} \backslash \mathcal{N}_{h}^{C C}\right) \cap \Gamma_{C}\left(\mathcal{N}_{h}^{C}\right.$ contains the nodes in $\Gamma_{C}$ which are not vertices of $\Omega$ ). For an element $K$, we will denote by $E_{K}$ the set of edges of $K$ and according to the above notation, we set $E_{K}^{i n t}=E_{K} \cap E_{h}^{i n t}, E_{K}^{N}=E_{K} \cap E_{h}^{N}, E_{K}^{C}=E_{K} \cap E_{h}^{C}$.

For an edge $E$ of an element $K$, introduce $\mathbf{n}_{K, E}=\left(n_{1}, n_{2}\right)$ the unit outward normal vector to $K$ along $E$ and the tangent vector $\mathbf{t}_{K, E}=\mathbf{n}_{K, E}^{\perp}=\left(-n_{2}, n_{1}\right)$. Furthermore for each edge $E$ we fix one of the two normal vectors and denote it by $\mathbf{n}_{E}$ and we set $\mathbf{t}_{E}=\mathbf{n}_{E}^{\perp}$. The jump of some vector valued function $\mathbf{v}$ across an edge $E \in E_{h}^{\text {int }}$ at a point $\mathbf{y} \in E$ is defined as

$$
\llbracket \mathbf{v}]]_{E}(\mathbf{y})=\lim _{\alpha \rightarrow+0} \mathbf{v}\left(\mathbf{y}+\alpha \mathbf{n}_{E}\right)-\mathbf{v}\left(\mathbf{y}-\alpha \mathbf{n}_{E}\right), \quad \forall E \in E_{h}^{i n t}
$$

Note that the sign of $[[\mathbf{v}]]_{E}$ depends on the orientation of $\mathbf{n}_{E}$. Finally we will need local subdomains (also called patches). As usual, let $\omega_{K}$ be the union of all elements having a nonempty intersection with $K$. Similarly for a node $\mathbf{x}$ and an edge $E$, let $\omega_{\mathbf{x}}=\cup_{K: \mathbf{x} \in K} K$ and $\omega_{E}=\cup_{\mathbf{x} \in \bar{E}} \omega_{\mathbf{x}}$.

The finite element space used in $\Omega$ is then defined by

$$
\mathbf{V}_{h}=\left\{\mathbf{v}_{h} \in(\mathcal{C}(\bar{\Omega}))^{2}: \quad \forall \kappa \in T_{h},\left.\quad \mathbf{v}_{h}\right|_{\kappa} \in\left(\mathbb{P}_{1}(\kappa)\right)^{2},\left.\quad \mathbf{v}_{h}\right|_{\Gamma_{D}}=\mathbf{0}\right\}
$$


We suppose that the contact area consists in several straight line segments, that we denote by $\overline{\Gamma_{C}^{i}}, 1 \leq i \leq q$ such that $\overline{\Gamma_{C}}=\cup_{i} \overline{\Gamma_{C}^{i}}$. In order to express the contact constraints by using Lagrange multipliers on the contact zone, we have to introduce the space

$$
W_{h}=\left\{\mu_{h}: \cup_{i} \overline{\Gamma_{C}^{i}} \rightarrow \mathbb{R},\left.\mu_{h}\right|_{\overline{\Gamma_{C}^{i}}} \in \mathcal{C}\left(\overline{\Gamma_{C}^{i}}\right), \exists \mathbf{v}_{h} \in \mathbf{V}_{h} \text { s.t. } \mathbf{v}_{h} \cdot \mathbf{n}=\mu_{h} \text { on } \cup_{i} \overline{\Gamma_{C}^{i}}\right\}
$$

The choice of the space $W_{h}$ allows us to define the following closed convex cone:

$$
M_{h}=\left\{\mu_{h} \in W_{h}: \int_{\Gamma_{C}} \mu_{h} \psi_{h} \mathrm{~d} \Gamma \geq 0, \forall \psi_{h} \in W_{h}, \psi_{h} \geq 0\right\} .
$$

Remark 3.1. It is easy to check that $M_{h} \not \subset M$.

The discretized mixed formulation of the unilateral contact problem without friction is to find $\mathbf{u}_{h} \in \mathbf{V}_{h}$ and $\lambda_{h} \in M_{h}$ satisfying:

$$
\left\{\begin{array}{l}
a\left(\mathbf{u}_{h}, \mathbf{v}_{h}\right)+b\left(\lambda_{h}, \mathbf{v}_{h}\right)=L\left(\mathbf{v}_{h}\right), \quad \forall \mathbf{v}_{h} \in \mathbf{V}_{h}, \\
b\left(\mu_{h}-\lambda_{h}, \mathbf{u}_{h}\right) \leq 0, \quad \forall \mu_{h} \in M_{h} .
\end{array}\right.
$$

Problem (10) consists in finding $\left(\lambda_{h}, \mathbf{u}_{h}\right) \in M_{h} \times \mathbf{V}_{h}$ satisfying

$$
\mathcal{L}\left(\mu_{h}, \mathbf{u}_{h}\right) \leq \mathcal{L}\left(\lambda_{h}, \mathbf{u}_{h}\right) \leq \mathcal{L}\left(\lambda_{h}, \mathbf{v}_{h}\right), \quad \forall \mathbf{v}_{h} \in \mathbf{V}_{h}, \forall \mu_{h} \in M_{h}
$$

where $\mathcal{L}\left(\mu_{h}, \mathbf{v}_{h}\right)=\frac{1}{2} a\left(\mathbf{v}_{h}, \mathbf{v}_{h}\right)-L\left(\mathbf{v}_{h}\right)+b\left(\mu_{h}, \mathbf{v}_{h}\right)$. In order to prove that there is a unique solution to problem (10), and since we are in the finite dimensional case, we only have to check (see [19], Thm. 3.9 and Ex. 3.8) that

$$
\sup _{\mathbf{v}_{h} \in \mathbf{V}_{h}, \mathbf{v}_{h} \neq \mathbf{0}} \frac{b\left(\mu_{h}, \mathbf{v}_{h}\right)}{\left\|\mathbf{v}_{h}\right\|_{1, \Omega}}
$$

is a norm on $W_{h}$. So we have to verify that

$$
\left\{\mu_{h} \in W_{h}: b\left(\mu_{h}, \mathbf{v}_{h}\right)=0, \forall \mathbf{v}_{h} \in \mathbf{V}_{h}\right\}=\{0\}
$$

which is satisfied according to the definition of $W_{h}$ in (9). As a consequence, we obtain the following statement:

Proposition 3.2. Problem (10) admits a unique solution $\left(\lambda_{h}, \mathbf{u}_{h}\right) \in M_{h} \times \mathbf{V}_{h}$.

Proposition 3.3. If $\left(\lambda_{h}, \mathbf{u}_{h}\right)$ is the solution of (10), then $\mathbf{u}_{h}$ is also the unique solution of the variational inequality: find $\mathbf{u}_{h}$ such that

$$
\mathbf{u}_{h} \in \mathbf{K}_{h}, \quad a\left(\mathbf{u}_{h}, \mathbf{v}_{h}-\mathbf{u}_{h}\right) \geq L\left(\mathbf{v}_{h}-\mathbf{u}_{h}\right), \quad \forall \mathbf{v}_{h} \in \mathbf{K}_{h},
$$

where $\mathbf{K}_{h}$ denotes the discrete closed convex cone of admissible displacement fields satisfying the non-penetration conditions, i.e.,

$$
\mathbf{K}_{h}=\left\{\mathbf{v}_{h} \in \mathbf{V}_{h}: \quad v_{h n} \leq 0 \text { on } \Gamma_{C}\right\} .
$$

Proof. Taking $\mu_{h}=0$ and $\mu_{h}=2 \lambda_{h}$ in (10) leads to $b\left(\lambda_{h}, \mathbf{u}_{h}\right)=0$ and to

$$
b\left(\mu_{h}, \mathbf{u}_{h}\right)=\int_{\Gamma_{C}} \mu_{h} u_{h n} \mathrm{~d} \Gamma \leq 0, \quad \forall \mu_{h} \in M_{h}
$$

The latter inequality implies by polarity that $u_{h n} \in-M_{h}^{*}$ (the notation $X^{*}$ stands for the positive polar cone of $X$ for the inner product on $W_{h}$ induced by $b(.,$.$) , see [22], p. 119). Let Q_{h}=\left\{\mu_{h} \in W_{h}: \mu_{h} \geq 0\right\}$. 
We have $M_{h}^{*}=\left(Q_{h}^{*}\right)^{*}=Q_{h}$ since $Q_{h}$ is a closed convex cone. Hence $u_{h n} \in-Q_{h}$ and $\mathbf{u}_{h} \in \mathbf{K}_{h}$. Besides (10) and $b\left(\lambda_{h}, \mathbf{u}_{h}\right)=0$ lead to

$$
a\left(\mathbf{u}_{h}, \mathbf{u}_{h}\right)=L\left(\mathbf{u}_{h}\right)
$$

and for any $\mathbf{v}_{h} \in \mathbf{K}_{h}$, we get

$$
a\left(\mathbf{u}_{h}, \mathbf{v}_{h}\right)-L\left(\mathbf{v}_{h}\right)=-b\left(\lambda_{h}, \mathbf{v}_{h}\right)=-\int_{\Gamma_{C}} \lambda_{h} v_{h n} \mathrm{~d} \Gamma \geq 0
$$

owing to $\lambda_{h} \in Q_{h}^{*}$.

Putting together (12) and (13) implies that $\mathbf{u}_{h}$ is solution of the variational inequality (11) which admits a unique solution according to Stampacchia's theorem.

Remark 3.4. A priori error analyses for this discretization of the unilateral contact problem can be found in $[4,12,23]$. The a priori error estimates are of order $h^{(1+\nu) / 2}$ if $\mathbf{u} \in\left(H^{3 / 2+\nu}(\Omega)\right)^{2}, 0<\nu \leq 1 / 2$ (see [4,12]). If an additional assumption dealing with the finiteness of transition points between contact and separation is added then an optimal error estimate of order $h^{1 / 2+\nu}$ is obtained (see [23]).

We consider the quasi-interpolation operator $\pi_{h}$ : for any $v \in L^{1}(\Omega)$, we define $\pi_{h} v$ as the unique element in $V_{h}=\left\{v_{h} \in \mathcal{C}(\bar{\Omega}): \forall \kappa \in T_{h},\left.v_{h}\right|_{\kappa} \in \mathbb{P}_{1}(\kappa),\left.v_{h}\right|_{\Gamma_{D}}=0\right\}$ such that:

$$
\pi_{h} v=\sum_{\mathbf{x} \in \mathcal{N}_{h} \backslash \mathcal{N}_{h}^{D}} \alpha_{\mathbf{x}}(v) \lambda_{\mathbf{x}}
$$

where for any $\mathbf{x} \in \mathcal{N}_{h} \backslash \mathcal{N}_{h}^{D}, \lambda_{\mathbf{x}}$ is the standard basis function in $V_{h}$ satisfying $\lambda_{\mathbf{x}}\left(\mathbf{x}^{\prime}\right)=\delta_{\mathbf{x}, \mathbf{x}^{\prime}}$, for all $\mathbf{x}^{\prime} \in \mathcal{N}_{h} \backslash \mathcal{N}_{h}^{D}$ and $\alpha_{\mathbf{x}}(v)$ is defined as follows:

$$
\alpha_{\mathbf{x}}(v)=\frac{1}{\left|\omega_{\mathbf{x}}\right|} \int_{\omega_{\mathbf{x}}} v(\mathbf{y}) \mathrm{d} \mathbf{y}, \quad \forall \mathbf{x} \in \mathcal{N}_{h} \backslash \mathcal{N}_{h}^{D}
$$

The following estimates hold (see, e.g., [29]):

Lemma 3.5. For any $v \in H_{\Gamma_{D}}^{1}(\Omega)$ we have

$$
\begin{aligned}
\left\|v-\pi_{h} v\right\|_{K} & \lesssim h_{K}\|\nabla v\|_{\omega_{K}}, \forall K \in T_{h}, \\
\left\|v-\pi_{h} v\right\|_{E} & \lesssim h_{E}^{1 / 2}\|\nabla v\|_{\omega_{E}}, \forall E \in E_{h} .
\end{aligned}
$$

Since we deal with vector valued functions we can define a vector valued operator (which we denote again by $\pi_{h}$ for the sake of simplicity) whose components are defined above. Consequently we can directly state the

Lemma 3.6. For any $\mathbf{v} \in \mathbf{V}$ we have

$$
\begin{aligned}
\left\|\mathbf{v}-\pi_{h} \mathbf{v}\right\|_{K} & \lesssim h_{K}\|\mathbf{v}\|_{1, \omega_{K}}, \forall K \in T_{h} \\
\left\|\mathbf{v}-\pi_{h} \mathbf{v}\right\|_{E} & \lesssim h_{E}^{1 / 2}\|\mathbf{v}\|_{1, \omega_{E}}, \forall E \in E_{h}
\end{aligned}
$$




\section{A first ERror estimator: $\eta$}

\subsection{Definition of the residual error estimators}

The element residual of the equilibrium equation (1) is defined by

$$
\operatorname{div} \boldsymbol{\sigma}\left(\mathbf{u}_{h}\right)+\mathbf{f}=\mathbf{f} \text { on } K
$$

As usual this element residual is replaced by some computable finite dimensional approximation called approximate element residual

$$
\mathbf{f}_{K} \in\left(\mathbb{P}_{k}(K)\right)^{2} \text {. }
$$

A current choice is to take $\mathbf{f}_{K}=\int_{K} \mathbf{f}(\mathbf{x}) /|K|$ since for $\mathbf{f} \in\left(H^{1}(\Omega)\right)^{2}$, scaling arguments yield $\left\|\mathbf{f}-\mathbf{f}_{K}\right\|_{K} \lesssim$ $h_{K}\|\mathbf{f}\|_{1, K}$ and is then negligible with respect to the estimator $\eta$ defined hereafter. In the same way $\mathbf{g}$ is approximated by a computable quantity denoted $\mathbf{g}_{E}$ on any $E \in E_{h}^{N}$.

Definition 4.1 (First residual error estimator). The local and global residual error estimators are defined by

$$
\begin{aligned}
\eta_{K} & =\left(\sum_{i=1}^{6} \eta_{i K}^{2}\right)^{1 / 2}, \\
\eta_{1 K} & =h_{K}\left\|\mathbf{f}_{K}\right\|_{K}, \\
\eta_{2 K} & =h_{K}^{1 / 2}\left(\sum_{E \in E_{K}^{i n t} \cup E_{K}^{N}}\left\|J_{E, n}\left(\mathbf{u}_{h}\right)\right\|_{E}^{2}\right)^{1 / 2}, \\
\eta_{3 K} & =h_{K}^{1 / 2}\left(\sum_{E \in E_{K}^{C}}\left\|\lambda_{h}+\sigma_{n}\left(\mathbf{u}_{h}\right)\right\|_{E}^{2}\right)^{1 / 2}, \\
\eta_{4 K} & =h_{K}^{1 / 2}\left(\sum_{E \in E_{K}^{C}}\left\|\sigma_{t}\left(\mathbf{u}_{h}\right)\right\|_{E}^{2}\right)^{1 / 2}, \\
\eta_{5 K} & =\left(\sum_{E \in E_{K}^{C}} \int_{E}-\lambda_{h+} u_{h n}\right)^{1 / 2}, \\
\eta_{6 K} & =\left(\sum_{E \in E_{K}^{C}}\left\|\lambda_{h-}\right\|_{E}^{2}\right)^{1 / 2}, \\
& =\left(\eta_{K}^{2}\right)^{1 / 2},
\end{aligned}
$$

where the notations $\lambda_{h+}$ and $\lambda_{h}$ - denote the positive and negative parts of $\lambda_{h}$, respectively; $J_{E, n}\left(\mathbf{u}_{h}\right)$ means the constraint jump of $\mathbf{u}_{h}$ in normal direction, i.e.,

$$
J_{E, n}\left(\mathbf{u}_{h}\right)=\left\{\begin{array}{l}
{\left[\left[\boldsymbol{\sigma}\left(\mathbf{u}_{h}\right) \mathbf{n}_{E}\right]_{E}, \forall E \in E_{h}^{i n t}\right.} \\
\boldsymbol{\sigma}\left(\mathbf{u}_{h}\right) \mathbf{n}_{E}-\mathbf{g}_{E}, \forall E \in E_{h}^{N}
\end{array}\right.
$$


The local and global approximation terms are defined by

$$
\zeta_{K}=\left(h_{K}^{2} \sum_{K^{\prime} \subset \omega_{K}}\left\|\mathbf{f}-\mathbf{f}_{K^{\prime}}\right\|_{K^{\prime}}^{2}+h_{E} \sum_{E \subset E_{K}^{N}}\left\|\mathbf{g}-\mathbf{g}_{E}\right\|_{E}^{2}\right)^{1 / 2}, \zeta=\left(\sum_{K \in T_{h}} \zeta_{K}^{2}\right)^{1 / 2} .
$$

Remark 4.2. In the Definition 4.1, we could also set instead of $\eta_{5 K}$ :

$$
\hat{\eta}_{5 K}=\left(\sum_{E \in E_{K}^{C}} \int_{E}-\lambda_{h-u_{h n}}\right)^{1 / 2}
$$

since $\int_{\Gamma_{C}} \lambda_{h+} u_{h n}=\int_{\Gamma_{C}} \lambda_{h-} u_{h n}$. Note that $\hat{\eta}_{5 K} \neq \eta_{5 K}$ although $\sum_{K \in T_{h}} \hat{\eta}_{5 K}^{2}=\sum_{K \in T_{h}} \eta_{5 K}^{2}$.

\subsection{Upper error bound}

Theorem 4.3. Let $(\lambda, \mathbf{u})$ be the solution of $(7)$ and let $\left(\lambda_{h}, \mathbf{u}_{h}\right)$ be the solution of (10). Then we have

$$
\left\|\mathbf{u}-\mathbf{u}_{h}\right\|_{1, \Omega}+\left\|\lambda-\lambda_{h}\right\|_{-\frac{1}{2}, \Gamma_{C}} \lesssim \eta+\zeta .
$$

Proof. Afterwards we adopt the following notation for the displacement error term:

$$
\mathbf{e}_{\mathbf{u}}=\mathbf{u}-\mathbf{u}_{h} .
$$

Let $\mathbf{v}_{h} \in \mathbf{V}_{h}$. From the $\mathbf{V}$-ellipticity of $a(.,$.$) and the equilibrium equations in (7) and (10) we obtain:$

$$
\begin{aligned}
\left\|\mathbf{e}_{\mathbf{u}}\right\|_{1, \Omega}^{2} & \lesssim a\left(\mathbf{u}-\mathbf{u}_{h}, \mathbf{u}-\mathbf{u}_{h}\right) \\
& =a\left(\mathbf{u}-\mathbf{u}_{h}, \mathbf{u}-\mathbf{v}_{h}\right)+a\left(\mathbf{u}-\mathbf{u}_{h}, \mathbf{v}_{h}-\mathbf{u}_{h}\right) \\
& =L\left(\mathbf{u}-\mathbf{v}_{h}\right)-b\left(\lambda, \mathbf{u}-\mathbf{v}_{h}\right)-a\left(\mathbf{u}_{h}, \mathbf{u}-\mathbf{v}_{h}\right)+b\left(\lambda_{h}-\lambda, \mathbf{v}_{h}-\mathbf{u}_{h}\right)
\end{aligned}
$$

Integrating by parts on each triangle $K$, using the definition of $J_{E, n}\left(\mathbf{u}_{h}\right)$ in (17) and the complementarity conditions $b(\lambda, \mathbf{u})=b\left(\lambda_{h}, \mathbf{u}_{h}\right)=0$ yields:

$$
\begin{aligned}
\left\|\mathbf{e}_{\mathbf{u}}\right\|_{1, \Omega}^{2} \lesssim & \int_{\Omega} \mathbf{f} \cdot\left(\mathbf{u}-\mathbf{v}_{h}\right)+b\left(\lambda_{h}, \mathbf{v}_{h}\right)+b\left(\lambda, \mathbf{u}_{h}\right)+\sum_{E \in E_{h}^{N}} \int_{E}\left(\mathbf{g}-\mathbf{g}_{E}\right) \cdot\left(\mathbf{u}-\mathbf{v}_{h}\right) \\
& -\sum_{E \in E_{h}^{C}} \int_{E}\left(\boldsymbol{\sigma}\left(\mathbf{u}_{h}\right) \mathbf{n}\right) \cdot\left(\mathbf{u}-\mathbf{v}_{h}\right)-\sum_{E \in E_{h}^{i n t} \cup E_{h}^{N}} \int_{E} J_{E, n}\left(\mathbf{u}_{h}\right) \cdot\left(\mathbf{u}-\mathbf{v}_{h}\right) .
\end{aligned}
$$

Splitting up the integrals on $\Gamma_{C}$ into normal and tangential components gives:

$$
\begin{aligned}
\left\|\mathbf{e}_{\mathbf{u}}\right\|_{1, \Omega}^{2} \lesssim & \int_{\Omega} \mathbf{f} \cdot\left(\mathbf{u}-\mathbf{v}_{h}\right)+b\left(\lambda_{h}, \mathbf{u}\right)+b\left(\lambda, \mathbf{u}_{h}\right) \\
& +\sum_{E \in E_{h}^{C}} \int_{E}\left(\lambda_{h}+\sigma_{n}\left(\mathbf{u}_{h}\right)\right)\left(v_{h n}-u_{n}\right)+\sum_{E \in E_{h}^{C}} \int_{E} \sigma_{t}\left(\mathbf{u}_{h}\right)\left(v_{h t}-u_{t}\right) \\
& -\sum_{E \in E_{h}^{\text {int }} \cup E_{h}^{N}} \int_{E} J_{E, n}\left(\mathbf{u}_{h}\right) \cdot\left(\mathbf{u}-\mathbf{v}_{h}\right)+\sum_{E \in E_{h}^{N}} \int_{E}\left(\mathbf{g}-\mathbf{g}_{E}\right) \cdot\left(\mathbf{u}-\mathbf{v}_{h}\right) .
\end{aligned}
$$


We now need to estimate each term of this right-hand side. For that purpose, we take

$$
\mathbf{v}_{h}=\mathbf{u}_{h}+\pi_{h}\left(\mathbf{u}-\mathbf{u}_{h}\right)
$$

where $\pi_{h}$ is the quasi-interpolation operator defined in Lemma 3.6.

We start with the integral term. Cauchy-Schwarz's inequality implies

$$
\left|\int_{\Omega} \mathbf{f} \cdot\left(\mathbf{u}-\mathbf{v}_{h}\right)\right| \leq \sum_{K \in T_{h}}\|\mathbf{f}\|_{K}\left\|\mathbf{u}-\mathbf{v}_{h}\right\|_{K},
$$

and it suffices to estimate $\left\|\mathbf{u}-\mathbf{v}_{h}\right\|_{K}$ for any triangle $K$. From the definition of $\mathbf{v}_{h}$ and (15) we get:

$$
\left\|\mathbf{u}-\mathbf{v}_{h}\right\|_{K}=\left\|\mathbf{e}_{\mathbf{u}}-\pi_{h} \mathbf{e}_{\mathbf{u}}\right\|_{K} \lesssim h_{K}\left\|\mathbf{e}_{\mathbf{u}}\right\|_{1, \omega_{K}}
$$

As a consequence

$$
\left|\int_{\Omega} \mathbf{f} \cdot\left(\mathbf{u}-\mathbf{v}_{h}\right)\right| \lesssim(\eta+\zeta)\left\|\mathbf{e}_{\mathbf{u}}\right\|_{1, \Omega} .
$$

We now consider the interior and Neumann boundary terms in (20): as previously the application of CauchySchwarz's inequality leads to

$$
\left|\sum_{E \in E_{h}^{i n t} \cup E_{h}^{N}} \int_{E} J_{E, n}\left(\mathbf{u}_{h}\right) \cdot\left(\mathbf{u}-\mathbf{v}_{h}\right)\right| \leq \sum_{E \in E_{h}^{i n t} \cup E_{h}^{N}}\left\|J_{E, n}\left(\mathbf{u}_{h}\right)\right\|_{E}\left\|\mathbf{u}-\mathbf{v}_{h}\right\|_{E} .
$$

Therefore using the expression (21) and estimate (16), we obtain

$$
\left\|\mathbf{u}-\mathbf{v}_{h}\right\|_{E}=\left\|\mathbf{e}_{\mathbf{u}}-\pi_{h} \mathbf{e}_{\mathbf{u}}\right\|_{E} \lesssim h_{E}^{1 / 2}\left\|\mathbf{e}_{\mathbf{u}}\right\|_{1, \omega_{E}}
$$

Inserting this estimate in the previous one we deduce that

$$
\left|\sum_{E \in E_{h}^{i n t} \cup E_{h}^{N}} \int_{E} J_{E, n}\left(\mathbf{u}_{h}\right) \cdot\left(\mathbf{u}-\mathbf{v}_{h}\right)\right| \lesssim \eta\left\|\mathbf{e}_{\mathbf{u}}\right\|_{1, \Omega}
$$

Moreover

$$
\left|\sum_{E \in E_{h}^{N}} \int_{E}\left(\mathbf{g}-\mathbf{g}_{E}\right) \cdot\left(\mathbf{u}-\mathbf{v}_{h}\right)\right| \lesssim \zeta\left\|\mathbf{e}_{\mathbf{u}}\right\|_{1, \Omega} .
$$

The two following terms are handled in a similar way as the previous ones so that

$$
\left|\sum_{E \in E_{h}^{C}} \int_{E}\left(\lambda_{h}+\sigma_{n}\left(\mathbf{u}_{h}\right)\right)\left(v_{h n}-u_{n}\right)\right| \lesssim \eta\left\|\mathbf{e}_{\mathbf{u}}\right\|_{1, \Omega}
$$

and

Noting that $u_{h n} \leq 0$ on $\Gamma_{C}$, we have

$$
\left|\sum_{E \in E_{h}^{C}} \int_{E} \sigma_{t}\left(\mathbf{u}_{h}\right)\left(v_{h t}-u_{t}\right)\right| \lesssim \eta\left\|\mathbf{e}_{\mathbf{u}}\right\|_{1, \Omega} .
$$

$$
b\left(\lambda, \mathbf{u}_{h}\right) \leq 0
$$


and it remains to estimate one term in (20). Using the discrete complementarity condition $b\left(\lambda_{h}, \mathbf{u}_{h}\right)=0$ implies

$$
\begin{aligned}
b\left(\lambda_{h}, \mathbf{u}\right)=\int_{\Gamma_{C}} \lambda_{h} u_{n} & =\int_{\Gamma_{C}} \lambda_{h}\left(u_{n}-u_{h n}\right) \\
& =\int_{\Gamma_{C}}\left(\lambda_{h+}-\lambda_{h-}\right)\left(u_{n}-u_{h n}\right) \\
& \leq-\int_{\Gamma_{C}} \lambda_{h+} u_{h n}-\int_{\Gamma_{C}} \lambda_{h-}\left(u_{n}-u_{h n}\right) \\
& \leq \eta^{2}-\int_{\Gamma_{C}} \lambda_{h-}\left(u_{n}-u_{h n}\right) .
\end{aligned}
$$

The last term in the previous expression is estimated using Cauchy-Schwarz's and Young's inequalities:

$$
\begin{aligned}
\left|\int_{\Gamma_{C}} \lambda_{h-}\left(u_{n}-u_{h n}\right)\right| & =\left|\sum_{E \in E_{h}^{C}} \int_{E} \lambda_{h-}\left(u_{n}-u_{h n}\right)\right| \\
& \leq \sum_{E \in E_{h}^{C}}\left\|\lambda_{h-}\right\| E\left\|u_{n}-u_{h n}\right\|_{E} \\
& \leq \sum_{E \in E_{h}^{C}}\left(\alpha\left\|u_{n}-u_{h n}\right\|_{E}^{2}+\frac{1}{4 \alpha}\left\|\lambda_{h-}\right\|_{E}^{2}\right),
\end{aligned}
$$

for any $\alpha>0$. A standard trace theorem implies the existence of $C>0$ such that

$$
\begin{aligned}
\left|\int_{\Gamma_{C}} \lambda_{h-}\left(u_{n}-u_{h n}\right)\right| & \leq \alpha\left\|u_{n}-u_{h n}\right\|_{\Gamma_{C}}^{2}+\frac{1}{4 \alpha} \sum_{E \in E_{h}^{C}}\left\|\lambda_{h-}\right\|_{E}^{2} \\
& \leq C \alpha\left\|\mathbf{e}_{\mathbf{u}}\right\|_{1, \Omega}^{2}+\frac{\eta^{2}}{4 \alpha}
\end{aligned}
$$

Estimates (28) and (29) give

$$
b\left(\lambda_{h}, \mathbf{u}\right) \leq C \alpha\left\|\mathbf{e}_{\mathbf{u}}\right\|_{1, \Omega}^{2}+\eta^{2}\left(1+\frac{1}{4 \alpha}\right)
$$

for any $\alpha>0$.

Putting together the estimates (22)-(27) and (30) with $\alpha$ small enough in (20), and using Young's inequality, we come to the conclusion that

$$
\left\|\mathbf{u}-\mathbf{u}_{h}\right\|_{1, \Omega} \lesssim \eta+\zeta .
$$

We now search for an upper bound on the discretization error $\lambda-\lambda_{h}$ corresponding to the multipliers. Let $\mathbf{v} \in \mathbf{V}$ and $\mathbf{v}_{h} \in \mathbf{V}_{h}$. From the equilibrium equations in (7) and (10) we get:

$$
\begin{aligned}
b\left(\lambda-\lambda_{h}, \mathbf{v}\right) & =b\left(\lambda, \mathbf{v}-\mathbf{v}_{h}\right)-b\left(\lambda_{h}, \mathbf{v}-\mathbf{v}_{h}\right)+b\left(\lambda-\lambda_{h}, \mathbf{v}_{h}\right) \\
& =L\left(\mathbf{v}-\mathbf{v}_{h}\right)-a\left(\mathbf{u}, \mathbf{v}-\mathbf{v}_{h}\right)-b\left(\lambda_{h}, \mathbf{v}-\mathbf{v}_{h}\right)+a\left(\mathbf{u}_{h}-\mathbf{u}, \mathbf{v}_{h}\right) \\
& =L\left(\mathbf{v}-\mathbf{v}_{h}\right)-a\left(\mathbf{u}-\mathbf{u}_{h}, \mathbf{v}\right)-a\left(\mathbf{u}_{h}, \mathbf{v}-\mathbf{v}_{h}\right)-b\left(\lambda_{h}, \mathbf{v}-\mathbf{v}_{h}\right)
\end{aligned}
$$


An integration by parts on each element $K$ gives

$$
\begin{aligned}
b\left(\lambda-\lambda_{h}, \mathbf{v}\right)= & \int_{\Omega} \mathbf{f} \cdot\left(\mathbf{v}-\mathbf{v}_{h}\right)-a\left(\mathbf{u}-\mathbf{u}_{h}, \mathbf{v}\right)-\sum_{E \in E_{h}^{i n t} \cup E_{h}^{N}} \int_{E} J_{E, n}\left(\mathbf{u}_{h}\right) \cdot\left(\mathbf{v}-\mathbf{v}_{h}\right) \\
& -\sum_{E \in E_{h}^{C}} \int_{E}\left(\lambda_{h}+\sigma_{n}\left(\mathbf{u}_{h}\right)\right)\left(v_{n}-v_{h n}\right)-\sum_{E \in E_{h}^{C}} \int_{E} \sigma_{t}\left(\mathbf{u}_{h}\right)\left(v_{t}-v_{h t}\right) \\
& +\sum_{E \in E_{h}^{N}} \int_{E}\left(\mathbf{g}-\mathbf{g}_{E}\right) \cdot\left(\mathbf{v}-\mathbf{v}_{h}\right) .
\end{aligned}
$$

Choosing $\mathbf{v}_{h}=\pi_{h} \mathbf{v}$ where $\pi_{h}$ is the quasi-interpolation operator defined in Lemma 3.6 and achieving a similar calculation as in $(22)-(26)$ we deduce that

$$
\left|b\left(\lambda-\lambda_{h}, \mathbf{v}\right)\right| \lesssim\left(\eta+\zeta+\left\|\mathbf{u}-\mathbf{u}_{h}\right\|_{1, \Omega}\right)\|\mathbf{v}\|_{1, \Omega}
$$

for any $\mathbf{v} \in \mathbf{V}$. As a consequence

$$
\left\|\lambda-\lambda_{h}\right\|_{-\frac{1}{2}, \Gamma_{C}} \lesssim \eta+\zeta+\left\|\mathbf{u}-\mathbf{u}_{h}\right\|_{1, \Omega}
$$

Putting together the two estimates (31) and (32) ends the proof of the theorem.

\subsection{Lower error bound}

Theorem 4.4. For all elements $K$, the following local lower error bounds hold:

$$
\begin{gathered}
\eta_{1 K} \lesssim\left\|\mathbf{u}-\mathbf{u}_{h}\right\|_{1, K}+\zeta_{K}, \\
\eta_{2 K} \lesssim\left\|\mathbf{u}-\mathbf{u}_{h}\right\|_{1, \omega_{K}}+\zeta_{K} .
\end{gathered}
$$

Assume that $\lambda \in L^{2}\left(\Gamma_{C}\right)$. For all elements $K$ such that $K \cap E_{h}^{C} \neq \emptyset$, the following local lower error bounds hold:

$$
\begin{aligned}
\eta_{3 K} & \lesssim \sum_{E \in E_{K}^{C}} h^{1 / 2}\left\|\lambda-\lambda_{h}\right\|_{E}+\left\|\mathbf{u}-\mathbf{u}_{h}\right\|_{1, K}+\zeta_{K}, \\
\eta_{4 K} & \lesssim\left\|\mathbf{u}-\mathbf{u}_{h}\right\|_{1, K}+\zeta_{K}, \\
\eta_{5 K} & \lesssim \sum_{E \in E_{K}^{C}}\left(\left\|\lambda-\lambda_{h}\right\|_{E}+\left\|\mathbf{u}-\mathbf{u}_{h}\right\|_{E}+\left\|\lambda-\lambda_{h}\right\|_{E}^{1 / 2}\left\|u_{n}\right\|_{E}^{1 / 2}+\left\|\mathbf{u}-\mathbf{u}_{h}\right\|_{E}^{1 / 2}\|\lambda\|_{E}^{1 / 2}\right), \\
\eta_{6 K} & \lesssim \sum_{E \in E_{K}^{C}}\left\|\lambda-\lambda_{h}\right\|_{E} .
\end{aligned}
$$

Proof. The estimates of $\eta_{1 K}$ and $\eta_{2 K}$ in (33) and (34) are standard (see, e.g., [28]).

We now estimate $\eta_{3 K}$. Writing $\mathbf{w}_{E}=w_{E n} \mathbf{n}+w_{E t} \mathbf{t}$ on $E \in E_{K}^{C}$ and denoting by $b_{E}$ the edge bubble function associated with $E$ (i.e., $b_{E}=4 \lambda_{a_{1}} \lambda_{a_{2}}$, when $a_{1}, a_{2}$ are the two extremities of $E$; we recall that $\lambda_{\mathbf{x}}$ is the standard basis function at node $\mathbf{x}$ in $V_{h}$ satisfying $\lambda_{\mathbf{x}}\left(\mathbf{x}^{\prime}\right)=\delta_{\mathbf{x}, \mathbf{x}^{\prime}}$ for any node $\mathbf{x}^{\prime}$, see (14)), we choose $w_{E n}=\left(\lambda_{h}+\sigma_{n}\left(\mathbf{u}_{h}\right)\right) b_{E}$ and $w_{E t}=0$ in the element $K$ containing $E$ (here we made a slight abuse of notation 
to simplify) and $\mathbf{w}_{E}=\mathbf{0}$ in $\bar{\Omega} \backslash K$. Therefore

$$
\begin{aligned}
\left\|\lambda_{h}+\sigma_{n}\left(\mathbf{u}_{h}\right)\right\|_{E}^{2} & \sim \int_{E}\left(\lambda_{h}+\sigma_{n}\left(\mathbf{u}_{h}\right)\right) w_{E n} \\
& =b\left(\lambda_{h}, \mathbf{w}_{E}\right)+\int_{K} \boldsymbol{\sigma}\left(\mathbf{u}_{h}\right): \varepsilon\left(\mathbf{w}_{E}\right) \\
& =b\left(\lambda_{h}, \mathbf{w}_{E}\right)-\int_{K} \boldsymbol{\sigma}\left(\mathbf{u}-\mathbf{u}_{h}\right): \varepsilon\left(\mathbf{w}_{E}\right)+\int_{K} \boldsymbol{\sigma}(\mathbf{u}): \varepsilon\left(\mathbf{w}_{E}\right) \\
& =b\left(\lambda_{h}-\lambda, \mathbf{w}_{E}\right)+L\left(\mathbf{w}_{E}\right)-\int_{K} \boldsymbol{\sigma}\left(\mathbf{u}-\mathbf{u}_{h}\right): \varepsilon\left(\mathbf{w}_{E}\right) \\
& \lesssim\left\|\lambda-\lambda_{h}\right\|_{E}\left\|\mathbf{w}_{E}\right\|_{E}+\|\mathbf{f}\|_{K}\left\|\mathbf{w}_{E}\right\|_{K}+\left\|\mathbf{u}-\mathbf{u}_{h}\right\|_{1, K}\left\|\mathbf{w}_{E}\right\|_{1, K} .
\end{aligned}
$$

An inverse inequality and estimate (33) imply

$$
\begin{aligned}
h_{K}^{1 / 2}\left\|\lambda_{h}+\sigma_{n}\left(\mathbf{u}_{h}\right)\right\|_{E} & \lesssim h^{1 / 2}\left\|\lambda-\lambda_{h}\right\|_{E}+\left\|\mathbf{u}-\mathbf{u}_{h}\right\|_{1, K}+h\|\mathbf{f}\|_{K} \\
& \lesssim h^{1 / 2}\left\|\lambda-\lambda_{h}\right\|_{E}+\left\|\mathbf{u}-\mathbf{u}_{h}\right\|_{1, K}+\zeta_{K} .
\end{aligned}
$$

This estimate gives the estimate of $\eta_{3 K}$ in (35). The bound of $\eta_{4 K}$ in (36) is obtained as previously by choosing $w_{E n}=0$ and $w_{E t}=\sigma_{t}\left(\mathbf{u}_{h}\right) b_{E}$.

We now consider $\eta_{5 K}$. If $E \in E_{K}^{C}$, let $F \subset E$ be the part of the edge where $\lambda_{h}=\lambda_{h+}$. So

$$
\begin{aligned}
\int_{E}-\lambda_{h+} u_{h n} & =\int_{F}-\lambda_{h} u_{h n} \\
& =\int_{F}\left(\lambda_{h}-\lambda\right)\left(u_{n}-u_{h n}\right)-\int_{F} \lambda_{h} u_{n}-\int_{F} \lambda u_{h n} \\
& =\int_{F}\left(\lambda_{h}-\lambda\right)\left(u_{n}-u_{h n}\right)-\int_{F}\left(\lambda_{h}-\lambda\right) u_{n}-\int_{F} \lambda\left(u_{h n}-u_{n}\right) \\
& \lesssim\left\|\lambda-\lambda_{h}\right\|_{E}\left\|\mathbf{u}-\mathbf{u}_{h}\right\|_{E}+\left\|\lambda-\lambda_{h}\right\|_{E}\left\|u_{n}\right\|_{E}+\left\|\mathbf{u}-\mathbf{u}_{h}\right\|_{E}\|\lambda\|_{E}
\end{aligned}
$$

The last estimate implies (37) by taking the square root.

The estimate on $\eta_{6 K}$ is obvious. Since $\lambda \geq 0$ then we have $0 \leq \lambda_{h-} \leq\left|\lambda-\lambda_{h}\right|$ on $\Gamma_{C}$. So

$$
\left\|\lambda_{h-}\right\|_{E} \leq\left\|\lambda-\lambda_{h}\right\|_{E}
$$

and (38) is proved.

Remark 4.5. Assume that $\mathbf{u} \in\left(H^{2}(\Omega)\right)^{2}$ (so $\lambda \in H^{\frac{1}{2}}\left(\Gamma_{C}\right)$ ), and that optimal a priori error estimates hold (note that the question of optimality remains open in the a priori error analysis since the known a priori error estimates are not optimal) and define:

$$
\eta_{i}=\left(\sum_{K \in T_{h}} \eta_{i K}^{2}\right)^{1 / 2}, \quad 1 \leq i \leq 6
$$

Then one would have $\eta_{1} \lesssim h, \eta_{2} \lesssim h, \eta_{3} \lesssim h, \eta_{4} \lesssim h, \eta_{5} \lesssim h^{1 / 4}, \eta_{6} \lesssim h^{1 / 2}$. So $\eta \lesssim h^{1 / 4}$.

\section{A SECOND ERROR ESTIMATOR: $\tilde{\eta}$}

The analysis of this error estimator requires a nonstandard definition of the error (in comparison with the already known results in the literature dealing with contact problems). We begin with some preliminaries. 


\subsection{Preliminaries}

For any $\mu_{h} \in W_{h}$ and $\mathbf{v}_{h} \in \mathbf{V}_{h}$ we define the bilinear form $c(.,$.$) such that$

$$
c\left(\mu_{h}, \mathbf{v}_{h}\right)=\sum_{i=1}^{q} \int_{\Gamma_{C}^{i}} I_{h}\left(\mu_{h} v_{h n}\right)
$$

where $I_{h}$ is the linear Lagrange interpolation operator at the nodes of $\overline{\Gamma_{C}^{i}}$ (to simplify the notations we write $I_{h}$ instead of $I_{h}^{i}$ ). Let $W_{h}^{+}$be the closed convex cone of nonnegative functions in $W_{h}$. We define the following discrete problem issued from (7): find $\mathbf{u}_{h} \in \mathbf{V}_{h}$ and $\tilde{\lambda}_{h} \in W_{h}^{+}$such that

$$
\left\{\begin{array}{l}
a\left(\mathbf{u}_{h}, \mathbf{v}_{h}\right)+c\left(\tilde{\lambda}_{h}, \mathbf{v}_{h}\right)=L\left(\mathbf{v}_{h}\right), \quad \forall \mathbf{v}_{h} \in \mathbf{V}_{h}, \\
c\left(\mu_{h}-\tilde{\lambda}_{h}, \mathbf{u}_{h}\right) \leq 0, \quad \forall \mu_{h} \in W_{h}^{+} .
\end{array}\right.
$$

The following proposition establishes the link between problems (39) and (10).

Proposition 5.1. (i) Problem (39) admits a unique solution $\left(\tilde{\lambda}_{h}, \mathbf{u}_{h}\right) \in W_{h}^{+} \times \mathbf{V}_{h}$.

(ii) The displacement field $\mathbf{u}_{h}$ in (39) coincides with the displacement field solving (10) (and also with the one solving (11)).

(iii) The link between the contact pressures $\tilde{\lambda}_{h}$ and $\lambda_{h}$ solving (39) and (10) is:

$$
c\left(\tilde{\lambda}_{h}, \mathbf{v}_{h}\right)=b\left(\lambda_{h}, \mathbf{v}_{h}\right), \quad \forall \mathbf{v}_{h} \in \mathbf{V}_{h}
$$

More precisely, if $\psi_{j}^{i}$ denotes the (scalar) canonical basis function at node $\mathbf{x}_{j}$ in $\overline{\Gamma_{C}^{i}} \backslash \overline{\Gamma_{D}}$, we have

$$
\tilde{\lambda}_{h}\left(\mathbf{x}_{j}\right)=\frac{\int_{\Gamma_{C}^{i}} \lambda_{h} \psi_{j}^{i}}{\int_{\Gamma_{C}^{i}} \psi_{j}^{i}}, \forall i, \forall j
$$

Proof. (i). As for problem (10) and according to [19] (Thm. 3.9 and Ex. 3.8), it suffices to verify that

$$
\sup _{\mathbf{v}_{h} \in \mathbf{V}_{h}, \mathbf{v}_{h} \neq \mathbf{0}} \frac{c\left(\mu_{h}, \mathbf{v}_{h}\right)}{\left\|\mathbf{v}_{h}\right\|_{1, \Omega}}
$$

is a norm on $W_{h}$, which reduces to the condition $\left\{\mu_{h} \in W_{h}: c\left(\mu_{h}, \mathbf{v}_{h}\right)=0, \forall \mathbf{v}_{h} \in \mathbf{V}_{h}\right\}=\{0\}$. The latter condition is fulfilled according to the definition of $W_{h}$ in (9).

(ii) The discussion is the same as in Proposition 3.3 noting that $c(.,$.$) induces an inner product on W_{h}$ and that $W_{h}^{+}$is a closed convex cone.

(iii) Equality (40) is straightforward. Let us show that $\operatorname{dim}\left(W_{h}\right)=\# \mathcal{N}_{h}^{C}+\# \mathcal{N}_{h}^{N C}+2 \# \mathcal{N}_{h}^{C C}$ where the notation \# stands for the cardinal of a set. We denote by $\psi_{j}^{i}$ the "canonical basis function" at node $\mathbf{x}_{j}$ in $\overline{\Gamma_{C}^{i}} \backslash \overline{\Gamma_{D}}$ (i.e., $\psi_{j}^{i}$ is defined on $\cup_{\ell} \overline{\Gamma_{C}^{\ell}}$, the support of $\psi_{j}^{i}$ lies in $\overline{\Gamma_{C}^{i}}, \psi_{j}^{i}$ is continuous and piecewise of degree one on $\overline{\Gamma_{C}^{i}}$, and $\left.\psi_{j}^{i}\left(\mathbf{x}_{k}\right)=\delta_{k, j}, \forall \mathbf{x}_{k} \in \overline{\Gamma_{C}^{i}} \backslash \overline{\Gamma_{D}}\right)$. Note that $\psi_{j}^{i}$ is not continuous if $\mathbf{x}_{j} \in \mathcal{N}_{h}^{C C}$.

If $\mathbf{x}_{j} \in \mathcal{N}_{h}^{C} \cup \mathcal{N}_{h}^{N C}$ it is straightforward that $\psi_{j}^{i} \in W_{h}$.

If $\mathbf{x}_{j} \in \mathcal{N}_{h}^{C C}$ then $\mathbf{x}_{j} \in \overline{\Gamma_{C}^{i}} \cup \overline{\Gamma_{C}^{i+1}}$. It suffices to show that there exists $\mathbf{v}_{h}=\left(v_{h 1}, v_{h 2}\right) \in \mathbf{V}_{h}$ such that $\psi_{j}^{i}=\mathbf{v}_{h} \cdot \mathbf{n}$ on $\cup_{\ell} \overline{\Gamma_{C}^{\ell}}$ and $\mathbf{w}_{h}=\left(w_{h 1}, w_{h 2}\right) \in \mathbf{V}_{h}$ such that $\psi_{j}^{i+1}=\mathbf{w}_{h} \cdot \mathbf{n}$ on $\cup_{\ell} \overline{\Gamma_{C}^{\ell}}$. Without loss of generality we can assume that the unit outward normal vector along $\Gamma_{C}^{i}$ is equal to $(0,-1)$ and then the unit outward normal vector along $\Gamma_{C}^{i+1}$ is equal to $(-\sin \theta, \cos \theta)$, for some $\left.\theta \in\right] 0,2 \pi[$ such that $\theta \neq \pi$ ( $\theta$ being the interior 
angle between $\Gamma_{C}^{i}$ and $\Gamma_{C}^{i+1}$ ). Since $\mathbf{v}_{h} \cdot \mathbf{n}$ is linear on $\Gamma_{C}^{i}$ and $\Gamma_{C}^{i+1}$, it suffices to show that $\mathbf{v}_{h} \cdot \mathbf{n}$ coincides with $\psi_{j}^{i}$ at the nodes. Therefore we take $\mathbf{v}_{h}$ equal to zero at each node except $\mathbf{x}_{j}$ and for the values at $\mathbf{x}_{j}$ we get

$$
\begin{aligned}
\mathbf{v}_{h}\left(\mathbf{x}_{j}\right) \cdot(0,-1) & =-v_{h 2}\left(\mathbf{x}_{j}\right)=1 \\
\mathbf{v}_{h}\left(\mathbf{x}_{j}\right) \cdot(-\sin \theta, \cos \theta) & =-v_{h 1}\left(\mathbf{x}_{j}\right) \sin \theta+v_{h 2}\left(\mathbf{x}_{j}\right) \cos \theta=0 .
\end{aligned}
$$

As $\sin \theta \neq 0$, this system has the unique solution: $v_{h 1}\left(\mathbf{x}_{j}\right)=-\cos \theta / \sin \theta$ and $v_{h 2}\left(\mathbf{x}_{j}\right)=-1$. The solution $\mathbf{w}_{h}$ is obtained similarly and is characterized by

$$
w_{h 1}\left(\mathbf{x}_{j}\right)=-\frac{1}{\sin \theta}, \quad w_{h 2}\left(\mathbf{x}_{j}\right)=0,
$$

$\mathbf{w}_{h}$ being zero at the other vertices.

Equality (41) follows from (40) and the previous discussion.

Remark 5.2. The a priori error estimates for the discretization (39) of the unilateral contact problem are given in [20]. The obtained estimates are the same as for the discretization (10) (see Rem. 3.4).

\subsection{Definition of the residual error estimators}

As for the first estimator the element residual is defined by $\operatorname{div} \boldsymbol{\sigma}\left(\mathbf{u}_{h}\right)+\mathbf{f}=\mathbf{f}$ on $K$ and this element residual is replaced by some computable finite dimensional approximation called approximate element residual: $\mathbf{f}_{K} \in\left(\mathbb{P}_{k}(K)\right)^{2}$. Similarly $\mathbf{g}$ is approximated by a computable quantity denoted $\mathbf{g}_{E}$ on any $E \in E_{h}^{N}$.

Definition 5.3 (Second residual error estimator). The local and global residual error estimators are defined by

$$
\begin{aligned}
\tilde{\eta}_{K} & =\left(\sum_{i=1}^{5} \tilde{\eta}_{i K}^{2}\right)^{1 / 2}, \\
\tilde{\eta}_{1 K} & =h_{K}\left\|\mathbf{f}_{K}\right\|_{K}, \\
\tilde{\eta}_{2 K} & =h_{K}^{1 / 2}\left(\sum_{E \in E_{K}^{i n t} \cup E_{K}^{N}}\left\|J_{E, n}\left(\mathbf{u}_{h}\right)\right\|_{E}^{2}\right)^{1 / 2}, \\
\tilde{\eta}_{3 K} & =h_{K}^{1 / 2}\left(\sum_{E \in E_{K}^{C}}\left\|\tilde{\lambda}_{h}+\sigma_{n}\left(\mathbf{u}_{h}\right)\right\|_{E}^{2}\right)^{1 / 2}, \\
\tilde{\eta}_{4 K} & =h_{K}^{1 / 2}\left(\sum_{E \in E_{K}^{C}}\left\|\sigma_{t}\left(\mathbf{u}_{h}\right)\right\|_{E}^{2}\right)^{1 / 2}, \\
\tilde{\eta}_{5 K} & =\left(\sum_{E \in E_{K}^{C}} \int_{E}-\tilde{\lambda}_{h} u_{h n}\right)^{1 / 2}, \\
\tilde{\eta} & =\left(\sum_{K \in T_{h}} \tilde{\eta}_{K}^{2}\right)^{1 / 2},
\end{aligned}
$$

where we recall that $J_{E, n}\left(\mathbf{u}_{h}\right)$ is the constraint jump of $\mathbf{u}_{h}$ in normal direction defined by (17). 
As in the previous section, the local and global approximation terms $\zeta_{K}$ and $\zeta$ are defined by (18).

Remark 5.4. From the previous definitions we have $\tilde{\eta}_{1 K}=\eta_{1 K}, \tilde{\eta}_{2 K}=\eta_{2 K}$ and $\tilde{\eta}_{4 K}=\eta_{4 K}$. We mention that there is no term as $\eta_{6}$ in the second estimator since the multipliers $\tilde{\lambda}_{h}$ are of constant sign (note that a similar approach was adopted in [27] for the obstacle problem).

\subsection{Upper error bound}

Theorem 5.5. Let $(\lambda, \mathbf{u})$ be the solution of $(7)$ and let $\left(\tilde{\lambda}_{h}, \mathbf{u}_{h}\right)$ be the solution of (39). We have

$$
\left\|\mathbf{u}-\mathbf{u}_{h}\right\|_{1, \Omega}+\left\|\lambda-\tilde{\lambda}_{h}\right\|_{-\frac{1}{2}, \Gamma_{C}} \lesssim \tilde{\eta}+\zeta
$$

Proof. We adopt the following notations for the error term in the displacement: $\mathbf{e}_{\mathbf{u}}=\mathbf{u}-\mathbf{u}_{h}$. Let $\mathbf{v}_{h} \in \mathbf{V}_{h}$. According to the $\mathbf{V}$-ellipticity of $a(.,$.$) and the equilibrium equations in (7) and (39) we obtain:$

$$
\begin{aligned}
\left\|\mathbf{e}_{\mathbf{u}}\right\|_{1, \Omega}^{2} \lesssim & a\left(\mathbf{u}-\mathbf{u}_{h}, \mathbf{u}-\mathbf{u}_{h}\right) \\
= & a\left(\mathbf{u}-\mathbf{u}_{h}, \mathbf{u}-\mathbf{v}_{h}\right)+a\left(\mathbf{u}-\mathbf{u}_{h}, \mathbf{v}_{h}-\mathbf{u}_{h}\right) \\
= & L\left(\mathbf{u}-\mathbf{v}_{h}\right)-b\left(\lambda, \mathbf{u}-\mathbf{v}_{h}\right)-a\left(\mathbf{u}_{h}, \mathbf{u}-\mathbf{v}_{h}\right)+b\left(\tilde{\lambda}_{h}-\lambda, \mathbf{v}_{h}-\mathbf{u}_{h}\right) \\
& +c\left(\tilde{\lambda}_{h}, \mathbf{v}_{h}-\mathbf{u}_{h}\right)-b\left(\tilde{\lambda}_{h}, \mathbf{v}_{h}-\mathbf{u}_{h}\right) .
\end{aligned}
$$

Integrating by parts on each triangle $K$, using the definition of $J_{E, n}\left(\mathbf{u}_{h}\right)$ in (17) and the complementarity conditions $b(\lambda, \mathbf{u})=b\left(\lambda_{h}, \mathbf{u}_{h}\right)=c\left(\tilde{\lambda}_{h}, \mathbf{u}_{h}\right)=0$ gives:

$$
\begin{aligned}
\left\|\mathbf{e}_{\mathbf{u}}\right\|_{1, \Omega}^{2} \lesssim & \int_{\Omega} \mathbf{f} \cdot\left(\mathbf{u}-\mathbf{v}_{h}\right)+b\left(\lambda, \mathbf{u}_{h}\right)+c\left(\tilde{\lambda}_{h}, \mathbf{v}_{h}\right)+\sum_{E \in E_{h}^{N}} \int_{E}\left(\mathbf{g}-\mathbf{g}_{E}\right) \cdot\left(\mathbf{u}-\mathbf{v}_{h}\right) \\
& -\sum_{E \in E_{h}^{C}} \int_{E}\left(\boldsymbol{\sigma}\left(\mathbf{u}_{h}\right) \mathbf{n}\right) \cdot\left(\mathbf{u}-\mathbf{v}_{h}\right)-\sum_{E \in E_{h}^{i n t} \cup E_{h}^{N}} \int_{E} J_{E, n}\left(\mathbf{u}_{h}\right) \cdot\left(\mathbf{u}-\mathbf{v}_{h}\right),
\end{aligned}
$$

which is the same inequality as in (19) according to (40). Splitting up the integrals on $\Gamma_{C}$ into normal and tangential components yields:

$$
\begin{aligned}
\left\|\mathbf{e}_{\mathbf{u}}\right\|_{1, \Omega}^{2} \lesssim & \int_{\Omega} \mathbf{f} \cdot\left(\mathbf{u}-\mathbf{v}_{h}\right)+b\left(\lambda, \mathbf{u}_{h}\right)+b\left(\tilde{\lambda}_{h}, \mathbf{u}\right)-b\left(\tilde{\lambda}_{h}, \mathbf{v}_{h}\right)+c\left(\tilde{\lambda}_{h}, \mathbf{v}_{h}\right) \\
& +\sum_{E \in E_{h}^{C}} \int_{E}\left(\tilde{\lambda}_{h}+\sigma_{n}\left(\mathbf{u}_{h}\right)\right)\left(v_{h n}-u_{n}\right)+\sum_{E \in E_{h}^{C}} \int_{E} \sigma_{t}\left(\mathbf{u}_{h}\right)\left(v_{h t}-u_{t}\right) \\
& -\sum_{E \in E_{h}^{\text {int }} \cup E_{h}^{N}} \int_{E} J_{E, n}\left(\mathbf{u}_{h}\right) \cdot\left(\mathbf{u}-\mathbf{v}_{h}\right)+\sum_{E \in E_{h}^{N}} \int_{E}\left(\mathbf{g}-\mathbf{g}_{E}\right) \cdot\left(\mathbf{u}-\mathbf{v}_{h}\right) .
\end{aligned}
$$

As before to estimate each term of this right-hand side, we take $\mathbf{v}_{h}$ of the form (21). 
We start with the integral term. As in the case of the first estimator we deduce from (21) and (15) that

$$
\begin{aligned}
\left|\int_{\Omega} \mathbf{f} \cdot\left(\mathbf{u}-\mathbf{v}_{h}\right)\right| & \leq \sum_{K \in T_{h}}\|\mathbf{f}\|_{K}\left\|\mathbf{u}-\mathbf{v}_{h}\right\|_{K} \\
& \lesssim \sum_{K \in T_{h}}\|\mathbf{f}\|_{K} h_{K}\left\|\mathbf{e}_{\mathbf{u}}\right\|_{1, \omega_{K}} \\
& \lesssim(\tilde{\eta}+\zeta)\left\|\mathbf{e}_{\mathbf{u}}\right\|_{1, \Omega} .
\end{aligned}
$$

We now consider the interior and Neumann boundary terms in (42): as previously the application of CauchySchwarz's inequality and (16) lead to

$$
\begin{aligned}
\left|\sum_{E \in E_{h}^{i n t} \cup E_{h}^{N}} \int_{E} J_{E, n}\left(\mathbf{u}_{h}\right) \cdot\left(\mathbf{u}-\mathbf{v}_{h}\right)\right| & \leq \sum_{E \in E_{h}^{i n t} \cup E_{h}^{N}}\left\|J_{E, n}\left(\mathbf{u}_{h}\right)\right\|_{E}\left\|\mathbf{u}-\mathbf{v}_{h}\right\|_{E} \\
& \lesssim \sum_{E \in E_{h}^{i n t} \cup E_{h}^{N}}\left\|J_{E, n}\left(\mathbf{u}_{h}\right)\right\|_{E} h_{E}^{1 / 2}\left\|\mathbf{e}_{\mathbf{u}}\right\|_{1, \omega_{E}} \\
& \lesssim \tilde{\eta}\left\|\mathbf{e}_{\mathbf{u}}\right\|_{1, \Omega} .
\end{aligned}
$$

Besides we get

$$
\left|\sum_{E \in E_{h}^{N}} \int_{E}\left(\mathbf{g}-\mathbf{g}_{E}\right) \cdot\left(\mathbf{u}-\mathbf{v}_{h}\right)\right| \lesssim \zeta\left\|\mathbf{e}_{\mathbf{u}}\right\|_{1, \Omega} .
$$

The two following terms are handled in a similar way as the previous ones so that

$$
\left|\sum_{E \in E_{h}^{C}} \int_{E}\left(\tilde{\lambda}_{h}+\sigma_{n}\left(\mathbf{u}_{h}\right)\right)\left(v_{h n}-u_{n}\right)\right| \lesssim \tilde{\eta}\left\|\mathbf{e}_{\mathbf{u}}\right\|_{1, \Omega}
$$

and

$$
\left|\sum_{E \in E_{h}^{C}} \int_{E} \sigma_{t}\left(\mathbf{u}_{h}\right)\left(v_{h t}-u_{t}\right)\right| \lesssim \tilde{\eta}\left\|\mathbf{e}_{\mathbf{u}}\right\|_{1, \Omega} .
$$

Noting that $u_{h n} \leq 0$ and $\tilde{\lambda}_{h} \geq 0$ on $\Gamma_{C}$, we have

$$
b\left(\lambda, \mathbf{u}_{h}\right) \leq 0, \quad b\left(\tilde{\lambda}_{h}, \mathbf{u}\right) \leq 0
$$

and it remains to estimate two terms in (42), namely

$$
\begin{aligned}
c\left(\tilde{\lambda}_{h}, \mathbf{v}_{h}\right)-b\left(\tilde{\lambda}_{h}, \mathbf{v}_{h}\right) & =-b\left(\tilde{\lambda}_{h}, \mathbf{u}_{h}\right)+c\left(\tilde{\lambda}_{h}, \pi_{h} \mathbf{e}_{\mathbf{u}}\right)-b\left(\tilde{\lambda}_{h}, \pi_{h} \mathbf{e}_{\mathbf{u}}\right) \\
& \leq \tilde{\eta}^{2}+\int_{\Gamma_{C}} I_{h}\left(\tilde{\lambda}_{h}\left(\pi_{h} \mathbf{e}_{\mathbf{u}}\right)_{n}\right)-\tilde{\lambda}_{h}\left(\pi_{h} \mathbf{e}_{\mathbf{u}}\right)_{n}
\end{aligned}
$$


The integral term in the previous expression is estimated as follows using a basic error estimate of numerical integration (trapezoidal formula):

$$
\begin{aligned}
\left|\int_{\Gamma_{C}} I_{h}\left(\tilde{\lambda}_{h}\left(\pi_{h} \mathbf{e}_{\mathbf{u}}\right)_{n}\right)-\tilde{\lambda}_{h}\left(\pi_{h} \mathbf{e}_{\mathbf{u}}\right)_{n}\right| & =\left|\sum_{E \in E_{h}^{C}} \int_{E} I_{h}\left(\tilde{\lambda}_{h}\left(\pi_{h} \mathbf{e}_{\mathbf{u}}\right)_{n}\right)-\tilde{\lambda}_{h}\left(\pi_{h} \mathbf{e}_{\mathbf{u}}\right)_{n}\right| \\
& \lesssim \sum_{E \in E_{h}^{C}} h_{E}^{3}\left|\left(\tilde{\lambda}_{h}\left(\pi_{h} \mathbf{e}_{\mathbf{u}}\right)_{n}\right)^{\prime \prime}\right| \\
& \lesssim \sum_{E \in E_{h}^{C}} h_{E}^{3}\left|\tilde{\lambda}_{h}^{\prime}\left(\left(\pi_{h} \mathbf{e}_{\mathbf{u}}\right)_{n}\right)^{\prime}\right| \\
& \lesssim \sum_{E \in E_{h}^{C}} h_{E}^{2}\left\|\tilde{\lambda}_{h}^{\prime}\right\|_{E}\left\|\left(\left(\pi_{h} \mathbf{e}_{\mathbf{u}}\right)_{n}\right)^{\prime}\right\|_{E} \\
& \lesssim \sum_{E \in E_{h}^{C}} h_{E}^{3 / 2}\left\|\tilde{\lambda}_{h}^{\prime}\right\|_{E}\left\|\pi_{h} \mathbf{e}_{\mathbf{u}}\right\|_{1, \omega_{E}} \\
& \lesssim \sum_{E \in E_{h}^{C}} h_{E}^{3 / 2}\left\|\tilde{\lambda}_{h}^{\prime}\right\|_{E}\left\|\mathbf{e}_{\mathbf{u}}\right\|_{1, \omega_{E}} \\
& =\sum_{E \in E_{h}^{C}} h_{E}^{3 / 2}\left\|\left(\tilde{\lambda}_{h}+\sigma_{n}\left(\mathbf{u}_{h}\right)\right)^{\prime}\right\|_{E}\left\|\mathbf{e}_{\mathbf{u}}\right\|_{1, \omega_{E}} \\
& \lesssim \sum_{E \in E_{h}^{C}} h_{E}^{1 / 2}\left\|\tilde{\lambda}_{h}+\sigma_{n}\left(\mathbf{u}_{h}\right)\right\|_{E}\left\|\mathbf{e}_{\mathbf{u}}\right\|_{1, \omega_{E}} \\
& \leq \tilde{\eta}\left\|\mathbf{e}_{\mathbf{u}}\right\|_{1, \Omega} .
\end{aligned}
$$

Above we have used the $H^{1}$ stability of $\pi_{h}$, proved in Lemma 3.1 of [8] (see also [28]) and the trace inequality on an element (see [28]).

Putting together the estimates (43)-(50) in (42) and using Young's inequality, we come to the conclusion that

$$
\left\|\mathbf{u}-\mathbf{u}_{h}\right\|_{1, \Omega} \lesssim \tilde{\eta}+\zeta
$$

Next we search for an upper bound on the discretization error $\lambda-\lambda_{h}$ corresponding to the multipliers. Let $\mathbf{v} \in \mathbf{V}$ and $\mathbf{v}_{h} \in \mathbf{V}_{h}$. From the equilibrium equations in (7) and (39) we get:

$$
\begin{aligned}
b\left(\lambda-\tilde{\lambda}_{h}, \mathbf{v}\right) & =b\left(\lambda, \mathbf{v}-\mathbf{v}_{h}\right)-b\left(\tilde{\lambda}_{h}, \mathbf{v}-\mathbf{v}_{h}\right)+b\left(\lambda-\lambda_{h}, \mathbf{v}_{h}\right)+b\left(\lambda_{h}-\tilde{\lambda}_{h}, \mathbf{v}_{h}\right) \\
& =L\left(\mathbf{v}-\mathbf{v}_{h}\right)-a\left(\mathbf{u}, \mathbf{v}-\mathbf{v}_{h}\right)-b\left(\tilde{\lambda}_{h}, \mathbf{v}-\mathbf{v}_{h}\right)+a\left(\mathbf{u}_{h}-\mathbf{u}, \mathbf{v}_{h}\right)+b\left(\lambda_{h}-\tilde{\lambda}_{h}, \mathbf{v}_{h}\right) \\
& =L\left(\mathbf{v}-\mathbf{v}_{h}\right)-a\left(\mathbf{u}-\mathbf{u}_{h}, \mathbf{v}\right)-a\left(\mathbf{u}_{h}, \mathbf{v}-\mathbf{v}_{h}\right)-b\left(\tilde{\lambda}_{h}, \mathbf{v}-\mathbf{v}_{h}\right)+b\left(\lambda_{h}-\tilde{\lambda}_{h}, \mathbf{v}_{h}\right) .
\end{aligned}
$$

An integration by parts on each element $K$ yields

$$
\begin{aligned}
b\left(\lambda-\tilde{\lambda}_{h}, \mathbf{v}\right)= & \int_{\Omega} \mathbf{f} \cdot\left(\mathbf{v}-\mathbf{v}_{h}\right)-a\left(\mathbf{u}-\mathbf{u}_{h}, \mathbf{v}\right)-\sum_{E \in E_{h}^{i n t} \cup E_{h}^{N}} \int_{E} J_{E, n}\left(\mathbf{u}_{h}\right) \cdot\left(\mathbf{v}-\mathbf{v}_{h}\right) \\
& -\sum_{E \in E_{h}^{C}} \int_{E}\left(\tilde{\lambda}_{h}+\sigma_{n}\left(\mathbf{u}_{h}\right)\right)\left(v_{n}-v_{h n}\right)-\sum_{E \in E_{h}^{C}} \int_{E} \sigma_{t}\left(\mathbf{u}_{h}\right)\left(v_{t}-v_{h t}\right) \\
& +c\left(\tilde{\lambda}_{h}, \mathbf{v}_{h}\right)-b\left(\tilde{\lambda}_{h}, \mathbf{v}_{h}\right)+\sum_{E \in E_{h}^{N}} \int_{E}\left(\mathbf{g}-\mathbf{g}_{E}\right) \cdot\left(\mathbf{v}-\mathbf{v}_{h}\right) .
\end{aligned}
$$


Choosing $\mathbf{v}_{h}=\pi_{h} \mathbf{v}$ where $\pi_{h}$ is the quasi-interpolation operator defined Lemma 3.6 and achieving a similar calculation as in (43)-(47) and (50) we deduce that

$$
\left|b\left(\lambda-\tilde{\lambda}_{h}, \mathbf{v}\right)\right| \lesssim\left(\tilde{\eta}+\zeta+\left\|\mathbf{u}-\mathbf{u}_{h}\right\|_{1, \Omega}\right)\|\mathbf{v}\|_{1, \Omega}
$$

for any $\mathbf{v} \in \mathbf{V}$. As a consequence

$$
\left\|\lambda-\tilde{\lambda}_{h}\right\|_{-\frac{1}{2}, \Gamma_{C}} \lesssim \tilde{\eta}+\zeta+\left\|\mathbf{u}-\mathbf{u}_{h}\right\|_{1, \Omega} .
$$

Putting together the two estimates (51) and (52) ends the proof of the theorem.

\subsection{Lower error bound}

Theorem 5.6. For all elements $K$, the following local lower error bounds hold:

$$
\begin{aligned}
\tilde{\eta}_{1 K} & \lesssim\left\|\mathbf{u}-\mathbf{u}_{h}\right\|_{1, K}+\zeta_{K}, \\
\tilde{\eta}_{2 K} & \lesssim\left\|\mathbf{u}-\mathbf{u}_{h}\right\|_{1, \omega_{K}}+\zeta_{K} .
\end{aligned}
$$

Assume that $\lambda \in L^{2}\left(\Gamma_{C}\right)$. For all elements $K$ such that $K \cap E_{h}^{C} \neq \emptyset$, the following local lower error bounds hold:

$$
\begin{aligned}
\tilde{\eta}_{3 K} & \lesssim \sum_{E \in E_{K}^{C}} h_{E}^{1 / 2}\left\|\lambda-\tilde{\lambda}_{h}\right\|_{E}+\left\|\mathbf{u}-\mathbf{u}_{h}\right\|_{1, K}+\zeta_{K} \\
\tilde{\eta}_{4 K} & \lesssim\left\|\mathbf{u}-\mathbf{u}_{h}\right\|_{1, K}+\zeta_{K} \\
\tilde{\eta}_{5 K} & \lesssim \tilde{\eta}_{3 K}^{1 / 2}\left\|\mathbf{u}_{h}\right\|_{1, K}^{1 / 2} .
\end{aligned}
$$

Proof. According to Remark 5.4 and Theorem 4.4 we only need to estimate $\tilde{\eta}_{3 K}$ and $\tilde{\eta}_{5 K}$.

We first estimate $\tilde{\eta}_{3 K}$. Writing $\mathbf{w}_{E}=w_{E n} \mathbf{n}+w_{E t} \mathbf{t}$ on $E \in E_{K}^{C}$ and denoting by $b_{E}$ the edge bubble function associated with $E$ (i.e., $b_{E}=4 \lambda_{a_{1}} \lambda_{a_{2}}$, when $a_{1}, a_{2}$ are the two extremities of $E$ ), we choose $w_{E n}=$ $\left(\tilde{\lambda}_{h}+\sigma_{n}\left(\mathbf{u}_{h}\right)\right) b_{E}$ and $w_{E t}=0$ in the element $K$ containing $E$ (here we made a slight abuse of notation to simplify) and $\mathbf{w}_{E}=\mathbf{0}$ in $\bar{\Omega} \backslash K$. So

$$
\begin{aligned}
\left\|\tilde{\lambda}_{h}+\sigma_{n}\left(\mathbf{u}_{h}\right)\right\|_{E}^{2} & \sim \int_{E}\left(\tilde{\lambda}_{h}+\sigma_{n}\left(\mathbf{u}_{h}\right)\right) w_{E n} \\
& =b\left(\tilde{\lambda}_{h}, \mathbf{w}_{E}\right)+\int_{K} \boldsymbol{\sigma}\left(\mathbf{u}_{h}\right): \boldsymbol{\varepsilon}\left(\mathbf{w}_{E}\right) \\
& =b\left(\tilde{\lambda}_{h}, \mathbf{w}_{E}\right)-\int_{K} \boldsymbol{\sigma}\left(\mathbf{u}-\mathbf{u}_{h}\right): \boldsymbol{\varepsilon}\left(\mathbf{w}_{E}\right)+\int_{K} \boldsymbol{\sigma}(\mathbf{u}): \boldsymbol{\varepsilon}\left(\mathbf{w}_{E}\right) \\
& =b\left(\tilde{\lambda}_{h}-\lambda, \mathbf{w}_{E}\right)+L\left(\mathbf{w}_{E}\right)-\int_{K} \boldsymbol{\sigma}\left(\mathbf{u}-\mathbf{u}_{h}\right): \boldsymbol{\varepsilon}\left(\mathbf{w}_{E}\right) \\
& \lesssim\left\|\lambda-\tilde{\lambda}_{h}\right\|_{E}\left\|_{\mathbf{w}_{E}}\right\|_{E}+\|\mathbf{f}\|_{K}\left\|\mathbf{w}_{E}\right\|_{K}+\left\|\mathbf{u}-\mathbf{u}_{h}\right\|_{1, K}\left\|\mathbf{w}_{E}\right\|_{1, K}
\end{aligned}
$$

An inverse inequality and estimate (53) imply

$$
\begin{aligned}
h_{K}^{1 / 2}\left\|\tilde{\lambda}_{h}+\sigma_{n}\left(\mathbf{u}_{h}\right)\right\|_{E} & \lesssim h^{1 / 2}\left\|\lambda-\tilde{\lambda}_{h}\right\|_{E}+\left\|\mathbf{u}-\mathbf{u}_{h}\right\|_{1, K}+h\|\mathbf{f}\|_{K} \\
& \lesssim h^{1 / 2}\left\|\lambda-\tilde{\lambda}_{h}\right\|_{E}+\left\|\mathbf{u}-\mathbf{u}_{h}\right\|_{1, K}+\zeta_{K} .
\end{aligned}
$$


This bound gives the estimate of $\tilde{\eta}_{3 K}$ in (54).

We finally consider $\tilde{\eta}_{5 K}$. If $E \in E_{K}^{C}$, one has

$$
\begin{aligned}
\int_{E}-\tilde{\lambda}_{h} u_{h n} & =\int_{E} I_{h}\left(\tilde{\lambda}_{h} u_{h n}\right)-\tilde{\lambda}_{h} u_{h n} \\
& \lesssim h_{E}^{3}\left|\left(\tilde{\lambda}_{h} u_{h n}\right)^{\prime \prime}\right| \\
& \lesssim h_{E}^{3}\left|\tilde{\lambda}_{h}^{\prime} u_{h n}^{\prime}\right| \\
& \lesssim h_{E}^{2}\left\|\tilde{\lambda}_{h}^{\prime}\right\|_{E}\left\|u_{h n}^{\prime}\right\|_{E} \\
& =h_{E}^{2}\left\|\left(\tilde{\lambda}_{h}+\sigma_{n}\left(\mathbf{u}_{h}\right)\right)^{\prime}\right\|_{E}\left\|u_{h n}^{\prime}\right\|_{E} \\
& \lesssim h_{E}\left\|\tilde{\lambda}_{h}+\sigma_{n}\left(\mathbf{u}_{h}\right)\right\|_{E}\left\|u_{h n}^{\prime}\right\|_{E} \\
& \lesssim h_{E}^{1 / 2} \tilde{\eta}_{3 K}\left\|u_{h n}^{\prime}\right\|_{E} \\
& \lesssim \tilde{\eta}_{3 K}\left\|\mathbf{u}_{h}\right\|_{1, K} .
\end{aligned}
$$

The last estimate implies (55) by taking the square root.

Remark 5.7. Assume that $\mathbf{u} \in\left(H^{2}(\Omega)\right)^{2}$ (so that $\lambda \in H^{\frac{1}{2}}\left(\Gamma_{C}\right)$ ). Then the integral term in $\tilde{\eta}_{5 K}$ can be bounded as follows:

$$
\begin{aligned}
\int_{E}-\tilde{\lambda}_{h} u_{h n} & =\int_{E} I_{h}\left(\tilde{\lambda}_{h} u_{h n}\right)-\tilde{\lambda}_{h} u_{h n} \\
& \lesssim h_{E}^{3}\left|\left(\tilde{\lambda}_{h} u_{h n}\right)^{\prime \prime}\right| \\
& \lesssim h_{E}^{3}\left|\tilde{\lambda}_{h}^{\prime} u_{h n}^{\prime}\right| \\
& =h_{E}^{2}\left\|\tilde{\lambda}_{h}^{\prime} u_{h n}^{\prime}\right\|_{L^{1}(E)} \\
& \leq h_{E}^{2}\left\|\tilde{\lambda}_{h}^{\prime}\left(u_{h n}^{\prime}-u_{n}^{\prime}\right)\right\|_{L^{1}(E)}+h_{E}^{2}\left\|\tilde{\lambda}_{h}^{\prime} u_{n}^{\prime}\right\|_{L^{1}(E)} \\
& \lesssim h_{E}^{2}\left\|\tilde{\lambda}_{h}^{\prime}\right\|_{E}\left\|\left(u_{h n}-u_{n}\right)^{\prime}\right\|_{E}+h_{E}^{2}\left\|\tilde{\lambda}_{h}^{\prime}\right\|_{L^{\frac{q}{q-1}}(E)}\left\|u_{n}^{\prime}\right\|_{L^{q}\left(\Gamma_{C}\right)} \\
& \lesssim h_{E}^{2}\left\|\left(\tilde{\lambda}_{h}+\sigma_{n}\left(\mathbf{u}_{h}\right)\right)^{\prime}\right\|_{E}\left\|\left(u_{h n}-u_{n}\right)^{\prime}\right\|_{E}+h_{E}^{2} \sqrt{q}\left\|\left(\tilde{\lambda}_{h}+\sigma_{n}\left(\mathbf{u}_{h}\right)\right)^{\prime}\right\|_{L^{\frac{q}{q-1}}(E)}\|\mathbf{u}\|_{2, \Omega} \\
& \lesssim h_{E}\left\|\tilde{\lambda}_{h}+\sigma_{n}\left(\mathbf{u}_{h}\right)\right\|_{E}\left\|\left(u_{h n}-u_{n}\right)^{\prime}\right\|_{E}+h_{E}^{2} \frac{q-2}{\frac{q-2}{2 q}} \sqrt{q}\left\|\left(\tilde{\lambda}_{h}+\sigma_{n}\left(\mathbf{u}_{h}\right)\right)^{\prime}\right\|_{E}\|\mathbf{u}\|_{2, \Omega} \\
& \lesssim h_{E}^{1 / 2} \tilde{\eta}_{3 K}\left\|u_{n}-u_{h n}\right\|_{1, E}+h_{E} \sqrt{-\ln \left(h_{E}\right)} \tilde{\eta}_{3 K}\|\mathbf{u}\|_{2, \Omega},
\end{aligned}
$$

where $1<q<\infty$ (we choose $q=-\ln \left(h_{E}\right), h_{E}$ is supposed small enough) and we have used the following embedding (see [3]): for any real number $p \in[1, \infty[$,

$$
\|v\|_{L^{p}\left(\Gamma_{C}\right)} \leq C \sqrt{p}\|v\|_{H^{\frac{1}{2}}\left(\Gamma_{C}\right)}, \quad \forall v \in H^{\frac{1}{2}}\left(\Gamma_{C}\right)
$$

where $C$ is independent of $p$. Define

$$
\tilde{\eta}_{i}=\left(\sum_{K \in T_{h}} \tilde{\eta}_{i K}^{2}\right)^{1 / 2}, \quad 1 \leq i \leq 5 .
$$

Assume that optimal a priori error estimates hold (this requires additional assumptions, see Rem. (5.2)). Then the previous result leads to the following bounds: $\tilde{\eta}_{i} \lesssim h, 1 \leq i \leq 4$ and $\tilde{\eta}_{5} \lesssim h^{3 / 4}(-\ln (h))^{1 / 4}$. Therefore $\tilde{\eta} \lesssim$ $h^{3 / 4}(-\ln (h))^{1 / 4}$. If we use only the known a priori error estimates (see Rem. (5.2)), we get: $\tilde{\eta}_{i} \lesssim h^{3 / 4}, 1 \leq i \leq 4$ and $\tilde{\eta}_{5} \lesssim h^{5 / 8}(-\ln (h))^{1 / 4}$. Therefore $\tilde{\eta} \lesssim h^{5 / 8}(-\ln (h))^{1 / 4}$ and our result is not far away from "optimality" since we have a loss of convergence of only $h^{1 / 8}(-\ln (h))^{-1 / 4}$. 
The second error estimator allows us to obtain improved upper and lower bounds of the error in comparison with the one in the previous section. Note that the definition of the discretization error was modified in this section where we compare $\left\|\mathbf{u}-\mathbf{u}_{h}\right\|_{1, \Omega}+\left\|\lambda-\tilde{\lambda}_{h}\right\|_{-\frac{1}{2}, \Gamma_{C}}$ with $\tilde{\eta}$ (whereas in the previous section we compare $\left\|\mathbf{u}-\mathbf{u}_{h}\right\|_{1, \Omega}+\left\|\lambda-\lambda_{h}\right\|_{-\frac{1}{2}, \Gamma_{C}}$ with $\left.\eta\right)$. The forthcoming numerical experiments will help us to compare the performances of both estimators and suggest us that $\tilde{\eta}$ is more appropriate than $\eta$ for the unilateral contact problem.

\section{NUMERICAL EXPERIMENTS}

This section is concerned with the numerical implementation of both estimators. We suppose that the bodies are homogeneous isotropic materials so that Hooke's law (2) becomes:

$$
\boldsymbol{\sigma}(\mathbf{u})=\frac{E \nu}{(1-2 \nu)(1+\nu)} \operatorname{tr}(\varepsilon(\mathbf{u})) \mathbf{I}+\frac{E}{1+\nu} \varepsilon(\mathbf{u})
$$

where I represents the identity matrix, $t r$ is the trace operator, $E$ and $\nu$ denote Young's modulus and Poisson's ratio, respectively with $E>0$ and $0 \leq \nu<1 / 2$.

Our main aim is to validate our theoretical results by computing the different contributions of the estimators $\eta$ and $\tilde{\eta}$ and their orders of convergence for different meshes. We also compute some effectivity indices and show that the estimator can be determined in more general cases than the theoretical framework. In our numerical experiments we do not consider optimized computations obtained from the estimators and a mesh adaptivity procedure which are beyond the scope of this paper. In the following we denote by $N_{C}$, the number of elements of the mesh on $\Gamma_{C}$. Since we use uniform meshes (made of triangular elements), this parameter measures the size of the mesh.

\subsection{First example: comparison of the error terms in both estimators}

We consider the domain $\Omega=] 0,1[\times] 0,1[$. We choose a realistic physical example. Namely we suppose that the body is an iron square of $1 \mathrm{~m}^{2}$ whose material characteristics are $E=2.1 \times 10^{11} \mathrm{~Pa}, \nu=0.3$ and $\rho=7800 \mathrm{~kg} \cdot \mathrm{m}^{-3}$. The body is clamped on $\left.\Gamma_{D}=\{1\} \times\right] 0,1\left[\right.$, it is initially in contact with $\left.\Gamma_{C}=\{0\} \times\right] 0,1[$ and it is acted on by its own weight only (with $\left.g=9.81 \mathrm{~m} \cdot \mathrm{s}^{-2}\right)$. Moreover $\left.\Gamma_{N}=\right] 0,1[\times(\{0\} \cup\{1\})$. We begin with achieving computations involving criss-cross meshes (this means that the body is divided into identical squares, each of them being divided into four identical triangles).

We observe (see Fig. 1) that $\Gamma_{C}$ is divided into two parts: an upper part where the body remains in contact with the axis $x=0$ and the lower part of $\Gamma_{C}$ where it separates from this axis. We determine the convergence of all the terms involved in both estimators $\eta$ and $\tilde{\eta}$ and we report the results in Tables 1 and 2 where we adopt the notations of Remarks 4.5 and 5.7:

$$
\eta_{i}=\left(\sum_{K \in T_{h}} \eta_{i K}^{2}\right)^{1 / 2}, 1 \leq i \leq 6, \quad \tilde{\eta}_{j}=\left(\sum_{K \in T_{h}} \tilde{\eta}_{j K}^{2}\right)^{1 / 2}, 1 \leq j \leq 5 .
$$

In these tables we compute the average convergence rates by averaging the rates between $N_{C}=4$ and $N_{C}=128$ and we give the limit rates if we observe that the rates seem to converge (this is not always the case). Note that the convergence rate of the terms: $\eta_{1}=\tilde{\eta}_{1}=h\left(\sum_{K \in T_{h}}\left\|\mathbf{f}_{K}\right\|_{K}^{2}\right)^{1 / 2} \sim h$ is 1 .

From the experiments we see that the terms $\eta_{2}=\tilde{\eta}_{2}, \eta_{3}, \tilde{\eta}_{3}$ and $\eta_{4}=\tilde{\eta}_{4}$ converge towards zero and that $\eta_{3}$ is close to $\tilde{\eta}_{3}\left(\eta_{2}\right.$ is the term converging the slowest towards zero and we observe that the main part of the error in $\eta$ and $\tilde{\eta}$ is located near the singular points $(1,0)$ and $(1,1))$. The error terms measuring the non fulfillment of the complementary condition (i.e., $\eta_{5}$ and $\tilde{\eta}_{5}$ ) show a convergence rate close to 1.5 which is much higher than the ones expected from the theoretical part (see Rems. 4.5 and 5.7). Moreover $\eta_{5}$ and $\tilde{\eta}_{5}$ are small in comparison with the other terms: this is not surprising since these terms are the only ones which depend on the Young modulus $E$. More precisely, if $\mathbf{u}(E)$ solves the elasticity problem in Figure 1 with a Young modulus $E$ 


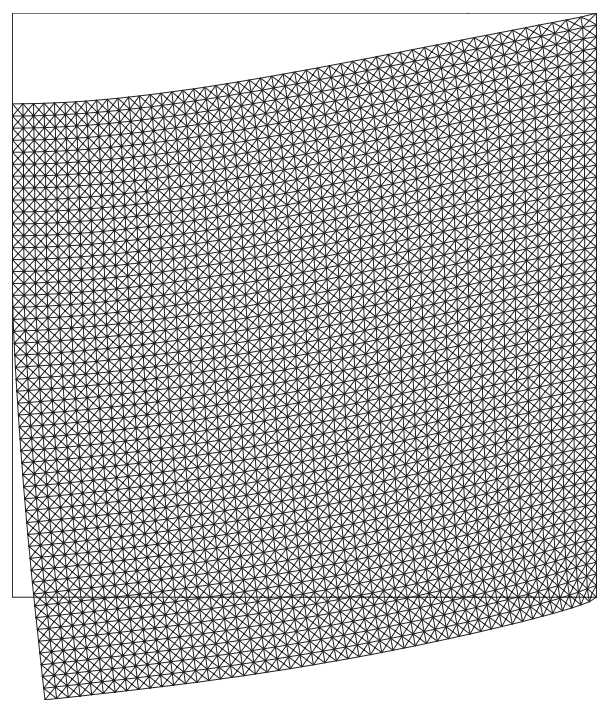

Figure 1. Initial and deformed configuration with $N_{C}=50$ (deformation is amplified by a factor $\left.2 \times 10^{5}\right)$.

TABle 1. Contributions in the estimator $\eta$ (criss-cross mesh).

\begin{tabular}{|c||c|c|c|c|c|}
\hline Estimator $\eta$ & $\eta_{2}$ & $\eta_{3}$ & $\eta_{4}$ & $\eta_{5}$ & $\eta_{6}$ \\
\hline \hline$N_{C}=1$ & 94716 & 35788 & 29586 & $3.20112 \times 10^{-2}$ & 13771 \\
\hline$N_{C}=2$ & 102670 & 23307 & 14128 & $1.96822 \times 10^{-2}$ & 12322 \\
\hline$N_{C}=4$ & 78460 & 9240.6 & 5989.7 & $5.02411 \times 10^{-3}$ & 820.96 \\
\hline$N_{C}=8$ & 50672 & 3150.8 & 2429.3 & $3.32207 \times 10^{-3}$ & 1562.1 \\
\hline$N_{C}=16$ & 30376 & 1108.1 & 938.10 & $4.31419 \times 10^{-4}$ & 53.836 \\
\hline$N_{C}=32$ & 17806 & 389.83 & 356.38 & $1.67433 \times 10^{-4}$ & 31.015 \\
\hline$N_{C}=64$ & 10424 & 138.49 & 134.19 & $6.30827 \times 10^{-5}$ & 17.193 \\
\hline$N_{C}=128$ & 6142.2 & 49.502 & 50.194 & $2.39545 \times 10^{-5}$ & 9.8331 \\
\hline \hline Convergence: & & & & & \\
Average rate & 0.74 & 1.51 & 1.38 & 1.54 & 1.28 \\
Limit rate & 0.76 & 1.48 & 1.42 & 1.40 & 0.81 \\
\hline
\end{tabular}

then it is easy to check from (56) that $\mathbf{u}(E) / k$ solves the same problem with a Young modulus $k E$ (if we had nonhomogeneous Dirichlet conditions, this would not be true) whereas $\boldsymbol{\sigma}(\mathbf{u}(E))=\boldsymbol{\sigma}(\mathbf{u}(k E))$. This implies that $\eta_{5}$ and $\tilde{\eta}_{5}$ behave as $E^{-1 / 2}$ and maybe a normalization of $\eta_{5}$ and $\tilde{\eta}_{5}$ would be necessary to avoid this phenomenon. The term $\eta_{6}$ whose theoretical convergence rate is also not optimal shows a non uniform decay towards zero, but faster than $\eta_{2}$. So we can reasonably expect that $\eta_{2}$ is the greatest term when $N_{C} \rightarrow+\infty$.

If we choose more general unstructured quasi-uniform meshes (instead of criss-cross meshes) on $\Omega$ we obtain the results reported in Tables 3 and 4 .

The main conclusions are similar to the ones when criss-cross meshes are used and we notice that the terms $\eta_{5}$ and $\eta_{6}$ converge rapidly but with a non uniform rate towards zero.

From this test we conclude that the implementation of $\tilde{\eta}$ is simpler than for $\eta$ : there is one term less in the computation of $\tilde{\eta}$ and the terms $\eta_{5}$ and $\eta_{6}$ involve negative parts of $\lambda_{h}$ (this would be more difficult to compute, 
TABLE 2. Contributions in the estimator $\tilde{\eta}$ (criss-cross mesh).

\begin{tabular}{|c||c|c|c|c|}
\hline Estimator $\tilde{\eta}$ & $\tilde{\eta}_{2}$ & $\tilde{\eta}_{3}$ & $\tilde{\eta}_{4}$ & $\tilde{\eta}_{5}$ \\
\hline \hline$N_{C}=1$ & 94716 & 11959 & 29586 & $3.39530 \times 10^{-2}$ \\
\hline$N_{C}=2$ & 102670 & 9536.6 & 14128 & $1.04538 \times 10^{-2}$ \\
\hline$N_{C}=4$ & 78460 & 5014.3 & 5989.7 & $6.93578 \times 10^{-3}$ \\
\hline$N_{C}=8$ & 50672 & 2270.7 & 2429.3 & $2.16306 \times 10^{-3}$ \\
\hline$N_{C}=16$ & 30376 & 900.17 & 938.10 & $7.61047 \times 10^{-4}$ \\
\hline$N_{C}=32$ & 17806 & 339.23 & 356.38 & $2.71989 \times 10^{-4}$ \\
\hline$N_{C}=64$ & 10424 & 125.04 & 134.19 & $9.69379 \times 10^{-5}$ \\
\hline$N_{C}=128$ & 6142.2 & 45.618 & 50.194 & $3.46036 \times 10^{-5}$ \\
\hline \hline Convergence: & & & & \\
Average rate & 0.74 & 1.36 & 1.38 & 1.53 \\
Limit rate & 0.76 & 1.45 & 1.42 & 1.49 \\
\hline
\end{tabular}

TABLE 3. Contributions in the estimator $\eta$ (unstructured mesh)

\begin{tabular}{|c||c|c|c|c|c|}
\hline Estimator $\eta$ & $\eta_{2}$ & $\eta_{3}$ & $\eta_{4}$ & $\eta_{5}$ & $\eta_{6}$ \\
\hline \hline$N_{C}=1$ & 88542 & 31184 & 32591 & $2.24674 \times 10^{-2}$ & 9851.5 \\
\hline$N_{C}=2$ & 99918 & 21238 & 13833 & $1.70126 \times 10^{-2}$ & 10315 \\
\hline$N_{C}=4$ & 95846 & 10861 & 5703.5 & $2.50567 \times 10^{-3}$ & 737.97 \\
\hline$N_{C}=8$ & 69987 & 4082.7 & 2448.9 & $3.27411 \times 10^{-3}$ & 1502.8 \\
\hline$N_{C}=16$ & 44123 & 1494.5 & 981.63 & $3.83538 \times 10^{-4}$ & 144.18 \\
\hline$N_{C}=32$ & 26776 & 505.22 & 373.78 & $1.0720 \times 10^{-4}$ & 42.542 \\
\hline$N_{C}=64$ & 15548 & 158.77 & 145.82 & $2.53311 \times 10^{-5}$ & 9.1921 \\
\hline$N_{C}=128$ & 9296.4 & 54.193 & 53.902 & $3.58675 \times 10^{-6}$ & 0.72587 \\
\hline \hline $\begin{array}{c}\text { Convergence: } \\
\text { Average rate } \\
\text { Limit rate }\end{array}$ & 0.67 & 1.53 & 1.35 & 1.89 & 2.00 \\
& 0.74 & 1.55 & 1.44 & no limit rate & no limit rate \\
\hline
\end{tabular}

especially in the three-dimensional case). Besides it seems that the convergence rate of $\tilde{\eta}_{5}$ is more uniform than the ones of $\eta_{5}$ and $\eta_{6}$ and that there are very few elements (near the transition points from contact to separation) where the error of $\tilde{\eta}_{5}$ is located and this is not the case for $\eta_{5}$ and $\eta_{6}$.

\subsection{Second example: a more regular case}

We consider the geometry $\hat{\Omega}=] 0,2[\times] 0,1[$ of area 2 square meters. We adopt symmetry conditions (i.e., $\left.u_{n}=0, \sigma_{t}(\mathbf{u})=0\right)$ on $\left.\Gamma_{S}=\{1\} \times\right] 0,1[$ and we achieve the computations on the square $\Omega=] 0,1[\times] 0,1[$. We set $\left.\Gamma_{C}=\right] 0,1\left[\times\{0\}\right.$ and $\Gamma_{N}$ is the remaining part of the boundary of $\Omega$. A Poisson ratio of $\nu=0.2$ and a Young modulus of $E=1 \mathrm{~Pa}$ are chosen (the latter value is of course not realistic from a physical point of view). A density of surface forces $\mathbf{g}$ of magnitude $1 \mathrm{~N} \cdot \mathrm{m}^{-2}$ oriented inwards $\Omega$ is applied on $\left.\{0\} \times\right] 1 / 2,1[$ and $] 1 / 2,1[\times\{1\}$. Such a configuration corresponds to a K-elliptic case (see [19], Thm. 6.3) and the problem admits a unique solution. We use criss-cross meshes in this example. Figure 2 depicts the initial and deformed configurations of the body. Here again $\Gamma_{C}$ shows a contact and a separation part. 
TABLE 4. Contributions in the estimator $\tilde{\eta}$ (unstructured mesh).

\begin{tabular}{|c||c|c|c|c|}
\hline Estimator $\tilde{\eta}$ & $\tilde{\eta}_{2}$ & $\tilde{\eta}_{3}$ & $\tilde{\eta}_{4}$ & $\tilde{\eta}_{5}$ \\
\hline \hline$N_{C}=1$ & 88542 & 19751 & 32591 & $2.38302 \times 10^{-2}$ \\
\hline$N_{C}=2$ & 99918 & 10687 & 13833 & $1.06738 \times 10^{-2}$ \\
\hline$N_{C}=4$ & 95846 & 7155.9 & 5703.5 & $5.95065 \times 10^{-3}$ \\
\hline$N_{C}=8$ & 69987 & 3387.7 & 2448.9 & $2.20742 \times 10^{-3}$ \\
\hline$N_{C}=16$ & 44123 & 1288.3 & 981.63 & $6.76565 \times 10^{-4}$ \\
\hline$N_{C}=32$ & 26776 & 453.06 & 373.78 & $2.45212 \times 10^{-4}$ \\
\hline$N_{C}=64$ & 15548 & 143.92 & 145.82 & $8.87555 \times 10^{-5}$ \\
\hline$N_{C}=128$ & 9296.4 & 49.590 & 53.902 & $3.17811 \times 10^{-5}$ \\
\hline \hline Convergence & & & & \\
Average rate & 0.67 & 1.43 & 1.35 & 1.51 \\
Limit rate & 0.74 & 1.54 & 1.44 & 1.48 \\
\hline
\end{tabular}

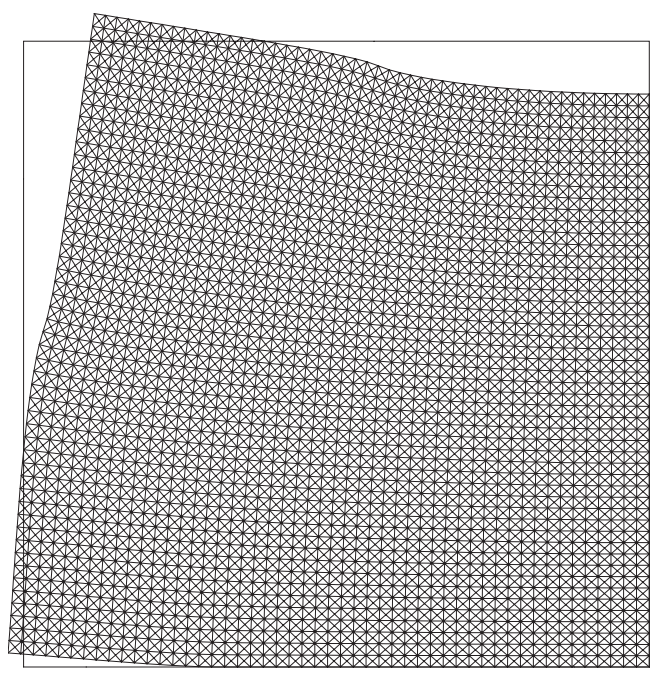

FiguRE 2. Initial and deformed configuration with $N_{C}=50$ (deformation is amplified by a factor 0.1 ).

It is easy to see that the symmetry conditions on $\Gamma_{S}$ lead to supplementary error terms similar to the ones in $\eta_{4}=\tilde{\eta}_{4}$ and we add these terms to $\eta_{2}$ and $\tilde{\eta}_{2}$. Moreover we have $\eta_{1}=\tilde{\eta}_{1}=0$. The results concerning both estimators are reported in Tables 5 and 6 .

As in the previous example, $\eta_{2}$ is the main term in the estimators with the lowest (but greater then in the previous example) convergence rate. We observe that the error is mainly located near the transition point between contact and separation and near the singularities $(0,1 / 2)$ and $(1 / 2,1)$ due to the jumps of the density of surface forces at these points and also, due to the very small Young modulus. As in the previous example the error terms $\eta_{5}$ and $\tilde{\eta}_{5}$ measuring the non fulfillment of the complementary condition converge with an higher rate than theoretically expected. The term $\eta_{6}$ shows a slow convergence rate in comparison with $\eta_{5}$ and $\tilde{\eta}_{5}$.

If we denote by $\left(a\left(\mathbf{u}-\mathbf{u}_{h}, \mathbf{u}-\mathbf{u}_{h}\right)\right)^{1 / 2}$ the energy norm of the discretization error (which is equivalent to the $\left(H^{1}(\Omega)\right)^{2}$ - norm of the error), we compute the convergence rates $\alpha, \tilde{\alpha}$ and $\beta$ of $\eta, \tilde{\eta}$ and $\left(a\left(\mathbf{u}-\mathbf{u}_{h}, \mathbf{u}-\mathbf{u}_{h}\right)\right)^{1 / 2}$ 
TABLE 5. Contributions in the estimator $\eta$.

\begin{tabular}{|c||c|c|c|c|c|}
\hline Estimator $\eta$ & $\eta_{2}$ & $\eta_{3}$ & $\eta_{4}$ & $\eta_{5}$ & $\eta_{6}$ \\
\hline \hline$N_{C}=2$ & 0.93715 & 0.16483 & $5.46634 \times 10^{-2}$ & $5.77944 \times 10^{-2}$ & $7.91410 \times 10^{-2}$ \\
\hline$N_{C}=4$ & 0.56976 & $7.17157 \times 10^{-2}$ & $3.63730 \times 10^{-2}$ & $2.32941 \times 10^{-2}$ & $4.26924 \times 10^{-2}$ \\
\hline$N_{C}=8$ & 0.33391 & $2.27644 \times 10^{-2}$ & $1.53814 \times 10^{-2}$ & $1.67857 \times 10^{-2}$ & $1.62697 \times 10^{-2}$ \\
\hline$N_{C}=16$ & 0.19152 & $1.00065 \times 10^{-2}$ & $7.79360 \times 10^{-3}$ & $4.73869 \times 10^{-3}$ & $7.69676 \times 10^{-3}$ \\
\hline$N_{C}=32$ & 0.10682 & $3.66909 \times 10^{-3}$ & $3.24986 \times 10^{-3}$ & $2.44547 \times 10^{-3}$ & $5.90999 \times 10^{-3}$ \\
\hline$N_{C}=64$ & $5.84672 \times 10^{-2}$ & $1.17072 \times 10^{-3}$ & $1.24725 \times 10^{-3}$ & $8.11375 \times 10^{-4}$ & $1.44793 \times 10^{-3}$ \\
\hline$N_{C}=128$ & $3.15747 \times 10^{-2}$ & $4.85373 \times 10^{-4}$ & $5.05263 \times 10^{-4}$ & $3.12555 \times 10^{-4}$ & $1.38974 \times 10^{-3}$ \\
\hline \hline Convergence: & & & & & \\
Average rate & 0.83 & 1.44 & 1.23 & 1.24 & 0.99 \\
Limit rate & 0.89 & 1.27 & 1.30 & 1.38 & no limit rate \\
\hline
\end{tabular}

TABLE 6. Contributions in the estimator $\tilde{\eta}$.

\begin{tabular}{|c||c|c|c|c|}
\hline Estimator $\tilde{\eta}$ & $\tilde{\eta}_{2}$ & $\tilde{\eta}_{3}$ & $\tilde{\eta}_{4}$ & $\tilde{\eta}_{5}$ \\
\hline \hline$N_{C}=2$ & 0.93715 & 0.10459 & $5.46634 \times 10^{-2}$ & $7.89660 \times 10^{-2}$ \\
\hline$N_{C}=4$ & 0.56976 & $5.01141 \times 10^{-2}$ & $3.63730 \times 10^{-2}$ & $1.41282 \times 10^{-2}$ \\
\hline$N_{C}=8$ & 0.33391 & $1.84373 \times 10^{-2}$ & $1.53814 \times 10^{-2}$ & $1.30765 \times 10^{-2}$ \\
\hline$N_{C}=16$ & 0.19152 & $7.52496 \times 10^{-3}$ & $7.79360 \times 10^{-3}$ & $2.79887 \times 10^{-3}$ \\
\hline$N_{C}=32$ & 0.10682 & $2.85827 \times 10^{-3}$ & $3.24986 \times 10^{-3}$ & $1.04594 \times 10^{-3}$ \\
\hline$N_{C}=64$ & $5.84672 \times 10^{-2}$ & $1.03522 \times 10^{-3}$ & $1.24725 \times 10^{-3}$ & $6.23388 \times 10^{-4}$ \\
\hline$N_{C}=128$ & $3.15747 \times 10^{-2}$ & $4.01120 \times 10^{-4}$ & $5.05263 \times 10^{-4}$ & $1.50507 \times 10^{-4}$ \\
\hline \hline Convergence: & & & & \\
Average rate & 0.83 & 1.39 & 1.23 & 1.31 \\
Limit rate & 0.89 & 1.37 & 1.30 & no limit rate \\
\hline
\end{tabular}

respectively. Moreover we are interested in determining the effectivity indices:

$$
\gamma=\frac{\eta}{\left(a\left(\mathbf{u}-\mathbf{u}_{h}, \mathbf{u}-\mathbf{u}_{h}\right)\right)^{1 / 2}} \quad \text { and } \quad \tilde{\gamma}=\frac{\tilde{\eta}}{\left(a\left(\mathbf{u}-\mathbf{u}_{h}, \mathbf{u}-\mathbf{u}_{h}\right)\right)^{1 / 2}} .
$$

These ratios measure the reliability of our estimators.

To our knowledge this problem does not admit an explicit solution $\mathbf{u}$. So, in order to determine $(a(\mathbf{u}-$ $\left.\left.\mathbf{u}_{h}, \mathbf{u}-\mathbf{u}_{h}\right)\right)^{1 / 2}$, we need to compute a reference solution denoted by $\mathbf{u}_{r e f}$ corresponding to a mesh which is as fine as possible. The most refined mesh corresponds to $N_{C}=128$ and it furnishes the reference solution $\mathbf{u}_{r e f}$ which is the chosen approximation for $\mathbf{u}$.

The results are reported in Table 7 where the errors are computed from $N_{C}=2$ to $N_{C}=32$ (the value $N_{C}=64$ would give a underestimated error in the energy norm since the field $\mathbf{u}_{h}$ is then too close to the reference solution). The average convergence rates (between $N_{C}=2$ and $N_{C}=32$ ) are the following: $\alpha=0.79$, $\tilde{\alpha}=0.79$ and $\beta=0.84$ and are therefore close. We also observe that the effectivity indices vary between 3.24 and 3.80 which correspond to reasonable values. 
TABLE 7. Estimators, error in the energy norm and effectivity indices.

\begin{tabular}{|c||c|c|c|c|c|}
\hline & $\eta$ & $\tilde{\eta}$ & $\left(a\left(\mathbf{u}-\mathbf{u}_{h}, \mathbf{u}-\mathbf{u}_{h}\right)\right)^{1 / 2}$ & $\gamma$ & $\tilde{\gamma}$ \\
\hline \hline$N_{C}=2$ & 0.95823 & 0.94784 & 0.29249 & 3.28 & 3.24 \\
\hline$N_{C}=4$ & 0.57746 & 0.57329 & 0.17068 & 3.38 & 3.36 \\
\hline$N_{C}=8$ & 0.33585 & 0.33503 & $9.66688 \times 10^{-2}$ & 3.47 & 3.47 \\
\hline$N_{C}=16$ & 0.19215 & 0.19185 & $5.32324 \times 10^{-2}$ & 3.61 & 3.61 \\
\hline$N_{C}=32$ & 0.10712 & 0.10691 & $2.81764 \times 10^{-2}$ & 3.80 & 3.79 \\
\hline
\end{tabular}

TABle 8. Contributions in the estimator $\tilde{\eta}$.

\begin{tabular}{|c||l|l|l|l|}
\hline Estimator $\tilde{\eta}$ & $\tilde{\eta}_{2}$ & $\tilde{\eta}_{3}$ & $\tilde{\eta}_{4}$ & $\tilde{\eta}_{5}$ \\
\hline \hline$h=1 / 10$ & 236.36 & 48.899 & 41.727 & 0.20734 \\
\hline$h=1 / 20$ & 138.60 & 17.341 & 20.835 & $6.25309 \times 10^{-2}$ \\
\hline$h=1 / 40$ & 89.959 & 10.645 & 10.912 & $2.98565 \times 10^{-2}$ \\
\hline$h=1 / 80$ & 42.857 & 2.2564 & 4.3787 & $1.68111 \times 10^{-2}$ \\
\hline$h=1 / 160$ & 22.865 & 1.1549 & 2.5559 & $4.50074 \times 10^{-3}$ \\
\hline \hline $\begin{array}{c}\text { Convergence: } \\
\text { Average rate }\end{array}$ & 0.84 & 1.35 & 1.00 & 1.38 \\
\hline
\end{tabular}

In this example the term $\eta_{6}$ converges slowly whereas the terms $\eta_{3}$ and $\tilde{\eta}_{3}, \eta_{5}$ and $\tilde{\eta}_{5}$ are similar. Concerning the convergence rates of $\eta$ and $\tilde{\eta}$ we find that there are similar and that the effectivity indices are also very close. So we conclude that there is no reason to choose $\eta$ instead of $\tilde{\eta}$ which is simpler to implement.

\subsection{Third example: a Hertz type problem}

In this test we consider the contact problem between an elastic disc (of $1 \mathrm{~m}$ in diameter) and a rigid half plane which corresponds to a Hertz type problem. A Poisson ratio of 0.4 and a Young modulus $E=10000 \mathrm{~Pa}$ are chosen. The aim of this example is to extend the range of applicability of the estimator $\tilde{\eta}$ to a more general case involving a curved contact zone with an initial gap between the foundation and the elastic body and an increasing contact area. Initially in the unconstrained configuration, the contact part between the disc and the half-plane is a single point. A density of surface loads $\mathrm{g}=(0,-200) \mathrm{N} \cdot \mathrm{m}^{-2}$ is applied on the upper quarter part of the boundary so that the problem becomes symmetric. We use quasi-uniform unstructured meshes. Note that the unilateral contact conditions in (5) have to be changed to take into account the gap between the contacting bodies. The conditions modelling unilateral contact on $\Gamma_{C}$ become:

$$
u_{n}-\xi \leq 0, \quad \sigma_{n}(\mathbf{u}) \leq 0, \quad \sigma_{n}(\mathbf{u})\left(u_{n}-\xi\right)=0,
$$

where $\xi=\xi(\mathbf{x})$ is the distance from $\mathbf{x} \in \Gamma_{C}$ to the rigid foundation. As a consequence the definition of $\tilde{\eta}_{5}$ in Definition 5.3 has to be changed into

$$
\tilde{\eta}_{5 K}=\left(\sum_{E \in E_{K}^{C}} \int_{E}-\tilde{\lambda}_{h}\left(u_{h n}-\xi\right)\right)^{1 / 2}
$$

The initial and a deformed configuration are depicted in Figure 3. The results concerning the implementation of $\tilde{\eta}$ are reported in Table 8 . 


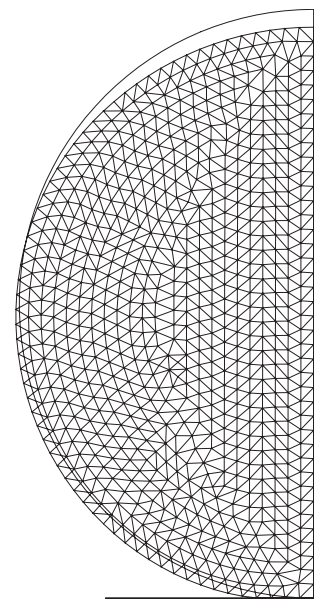

FiguRE 3. Initial and deformed configuration (deformation is not amplified).

As previously explained and observed in the first example, the term $\tilde{\eta}_{5}$ is small and it admits a convergence rate which is really more important than theoretically expected. We obtain for this example results which are similar to the previous ones.

\section{Conclusion}

In this work we propose and analyze two estimators ( $\eta$ and $\tilde{\eta}$ ) of residual type associated with two mixed finite element approximations of the two-dimensional frictionless unilateral contact problem in linear elasticity. For both estimators we obtain upper and lower bounds of the discretization error. From the numerical experiments we come to the conclusion that the results given by the two estimators are roughly speaking similar and that all the terms converge towards zero with satisfactory convergence rates (the slowest convergence is observed for the classical terms denoted by $\eta_{2}$ and $\tilde{\eta}_{2}$ which measure in particular the constraint jumps across the interior edges, and all the terms coming from the contact approximation converge better). Nevertheless $\tilde{\eta}$ has one term less to evaluate in comparison with $\eta$ and its numerical implementation is (a bit) simpler. We also observe that the supplementary term in $\eta\left(\right.$ i.e., $\left.\eta_{6}\right)$ does not admit in the general case a uniform convergence rate. Our conclusion concerning the comparison of both estimators is that $\tilde{\eta}$ could be more promising than $\eta$. Besides we see that the error terms measuring the non fulfillment of the complementarity condition (i.e., $\eta_{5}$ and $\tilde{\eta}_{5}$ ) converge much faster than theoretically expected; this allows us to expect that some improved theoretical estimates for these terms could be obtained.

Acknowledgements. This work is partially supported by "l'Agence Nationale de la Recherche", project ANR-05-JCJC0182-01.

\section{REFERENCES}

[1] R.A. Adams, Sobolev spaces. Academic Press (1975).

[2] K. Atkinson and W. Han, Theoretical numerical analysis: a functional analysis framework, in Texts in Applied Mathematics 39, Springer, New-York (2001); (second edition 2005).

[3] F. Ben Belgacem, Numerical simulation of some variational inequalities arisen from unilateral contact problems by the finite element method. SIAM J. Numer. Anal. 37 (2000) 1198-1216.

[4] F. Ben Belgacem and Y. Renard, Hybrid finite element methods for the Signorini problem. Math. Comp. 72 (2003) 1117-1145.

[5] V. Bostan, W. Han and B.D. Reddy, A posteriori error estimation and adaptive solution of elliptic variational inequalities of the second kind. Appl. Numer. Math. 52 (2005) 13-38. 
[6] D. Braess, A posteriori error estimators for obstacle problems - another look. Numer. Math. 101 (2005) 415-421.

[7] C. Carstensen, O. Scherf and P. Wriggers, Adaptive finite elements for elastic bodies in contact. SIAM J. Sci. Comput. 20 (1999) 1605-1626.

[8] Z. Chen and R.H. Nochetto, Residual type a posteriori error estimates for elliptic obstacle problems. Numer. Math. 84 (2000) $527-548$.

[9] P.G. Ciarlet, The finite element method for elliptic problems, in Handbook of Numerical Analysis, Volume II, Part 1, P.G. Ciarlet and J.-L. Lions Eds., North Holland (1991) 17-352.

[10] P. Coorevits, P. Hild and J.-P. Pelle, A posteriori error estimation for unilateral contact with matching and nonmatching meshes. Comput. Methods Appl. Mech. Engrg. 186 (2000) 65-83.

[11] P. Coorevits, P. Hild and M. Hjiaj, A posteriori error control of finite element approximations for Coulomb's frictional contact. SIAM J. Sci. Comput. 23 (2001) 976-999.

[12] P. Coorevits, P. Hild, K. Lhalouani and T. Sassi, Mixed finite element methods for unilateral problems: convergence analysis and numerical studies. Math. Comp. 71 (2002) 1-25.

[13] G. Duvaut and J.-L. Lions, Les inéquations en mécanique et en physique. Dunod (1972).

[14] C. Eck and W. Wendland, A residual-based error estimator for BEM-discretizations of contact problems. Numer. Math. 95 (2003) 253-282.

[15] G. Fichera, Problemi elastici con vincoli unilaterali il problema di Signorini con ambigue condizioni al contorno. Mem. Accad. Naz. Lincei. 8 (1964) 91-140.

[16] G. Fichera, Existence theorems in elasticity, in Handbuch der Physik, Band VIa/2, Springer (1972) $347-389$.

[17] R. Glowinski, Lectures on numerical methods for nonlinear variational problems, in Lectures on Mathematics and Physics 65, Notes by M. G. Vijayasundaram and M. Adimurthi, Tata Institute of Fundamental Research, Bombay; Springer-Verlag, Berlin-New York (1980).

[18] W. Han and M. Sofonea, Quasistatic contact problems in viscoelasticity and viscoplasticity. American Mathematical Society (2002).

[19] J. Haslinger, I. Hlaváček and J. Nečas, Numerical methods for unilateral problems in solid mechanics, in Handbook of Numerical Analysis, Volume IV, Part 2, P.G. Ciarlet and J.-L. Lions Eds., North Holland (1996) 313-485.

[20] P. Hild, A priori error analysis of a sign preserving mixed finite element method for contact problems. Preprint $2006 / 33$ of the Laboratoire de Mathématiques de Besançon, submitted.

[21] P. Hild and S. Nicaise, A posteriori error estimations of residual type for Signorini's problem. Numer. Math. 101 (2005) $523-549$.

[22] J.-B. Hiriart-Urruty and C. Lemaréchal, Convex analysis and minimization algorithms I. Springer (1993).

[23] S. Hüeber and B. Wohlmuth, An optimal error estimate for nonlinear contact problems. SIAM J. Numer. Anal. 43 (2005) $156-173$.

[24] N. Kikuchi and J.T. Oden, Contact problems in elasticity. SIAM (1988).

[25] T. Laursen, Computational contact and impact mechanics. Springer (2002).

[26] C.Y. Lee and J.T. Oden, A posteriori error estimation of $h$ - $p$ finite element approximations of frictional contact problems. Comput. Methods Appl. Mech. Engrg. 113 (1994) 11-45.

[27] A. Veeser, Efficient and reliable a posteriori error estimators for elliptic obstacle problems. SIAM J. Numer. Anal. 39 (2001) $146-167$.

[28] R. Verfürth, A review of a posteriori error estimation and adaptive mesh-refinement techniques. Wiley and Teubner (1996).

[29] R. Verfürth, A review of a posteriori error estimation techniques for elasticity problems. Comput. Methods Appl. Mech. Engrg. 176 (1999) 419-440.

[30] P. Wriggers, Computational Contact Mechanics. Wiley (2002).

[31] P. Wriggers and O. Scherf, Different a posteriori error estimators and indicators for contact problems. Mathl. Comput. Modelling 28 (1998) 437-447. 